\title{
Separable Kripke structures are algebraically universal
}

\author{
M. C. PINTO*
}

\begin{abstract}
For every poset $(I, \leq)$ and every family $\left(G_{i}\right)_{i \in I}$ of groups there exists a family of separable Kripke structures $\left(K_{i}\right)_{i \in I}$ on the same set, such that $G_{i} \cong \operatorname{Aut}\left(K_{i}\right)$ and $K_{i}$ is subalgebra of $K_{j}$ iff $i \leq j$, for $i, j \in I$. More generally, this work is concerned with representations of algebraic categories by means of the category of separable Kripke structures. Consequences thereof are mentioned. Thus, in contrast to the algebraic non-universality of the category of Boolean algebras we conclude the algebraic universality of the category of separable dynamic algebras. Perfect classes of Kripke structures are introduced.
\end{abstract}

\section{Introduction}

\subsection{On Kripke structures and dynamic algebras}

Dynamic algebras were introduced by Kozen [19] and Pratt [29] to provide models of Propositional Dynamic Logic (PDL). For relation to Computer Science and examples see [29].

Following [29] a dynamic algebra is a two-sorted algebra $(\mathcal{B}, \mathcal{A},<>)$ where

$$
\mathcal{B}=(B, \vee, \neg, 0) \text { and } \mathcal{A}=(A, ;, \cup, *)
$$

are one-sorted algebras with

$0: \quad 0$-ary operation (constant), $\neg, *: \quad 1$-ary operations,

$\vee, ;, \cup: \quad$ 2-ary operations,

and an operation named diamond $\quad<\cdot, \cdot>: A \times B \rightarrow B:$ mixed operation, satisfying the following conditions (where ";" is omitted, for brevity):

(i) $\mathcal{B}$ is a Boolean algebra;

(ii) $\langle a, 0>=0$ and $<a, p \vee q>=<a, p>\vee<a, q>$;

(iii) $\langle a \cup b, p>=<a, p>\vee<b, p>$;

(iv) $\langle a b, p\rangle=\langle a,\langle b, p\rangle\rangle$;

(v) $p \vee<a a^{*}, p>\leq<a^{*}, p>\leq p \vee<a^{*}, \neg p \wedge<a, p \gg$

for $a, b \in A$ and $p, q \in B$.

Obs.: $(p \leq q)$ is abbreviation of $(p \wedge q=p)$ and $(p \wedge q)$ is abbreviation of $(\neg(\neg p \vee \neg q))$.

Homomorphisms of dynamic algebras $h:(\mathcal{B}, \mathcal{A},<>) \longrightarrow\left(\mathcal{B}^{\prime}, \mathcal{A}^{\prime},<>\right)$ are defined in the usual way, as homomorphisms of two-sorted algebras, i.e., $h=\left(h_{1}, h_{2}\right)$ with $h_{1}: \mathcal{B} \longrightarrow \mathcal{B}^{\prime}$,

${ }^{*}$ Research supported partially by JNICT (Programa de Formação e Mobilidade de Recursos Humanos BD 1298 and Cultural Agreement between Portugal and Czech Republic), Instituto de Telecomunicações and Centro de Matemática da Universidade de Coimbra.

1991 Mathematics Subject Classification: 18B15, 06E25.

Keywords: Kripke structures, perfect class of Kripke structures, dynamic algebras, algebraic universality. 
$h_{2}: \mathcal{A} \longrightarrow \mathcal{A}^{\prime}$ such that $h_{1}$ and $h_{2}$ preserve the mentioned one-sorted operations and, moreover, $h$ preserves the mixed operation, that is, $\left.h_{1}(<a, p\rangle\right)=\left\langle h_{2}(a), h_{1}(p)\right\rangle$ for every $a \in \mathcal{A}$ and every $p \in \mathcal{B}$. Let $D A$ denote the category of all dynamic algebras (whenever we consider a category mentioning only its objects, we assume that its morphisms are all the homomorphisms among those objects).

A dynamic algebra $(\mathcal{B}, \mathcal{A},<>)$ is said to be separable if for all $a, b \in \mathcal{A}$,

$$
<a, p>=<b, p>\text { for every } p \in \mathcal{B} \quad \Longrightarrow \quad a=b .
$$

We denote by $S D A$ the full subcategory of $D A$ whose objects are the separable dynamic algebras.

Kripke structures, the traditional models of PDL, were presented in [29] as examples of dynamic algebras. They are defined as follows. The full Kripke structure on a given nonempty set $S$ is the pair $(\exp (S), \exp (S \times S))$ where $\exp (S)$ is the Boolean algebra of all subsets of $S$ (with the usual set theoretical operations) and $\exp (S \times S)$ is the set of all binary relations on $S$. The operations ; $\cup$ and $*$ on $\exp (S \times S)$ are the composition, the union and the reflective-transitive closure of binary relations, respectively. The reflective-transitive closure of $a \in \exp (S \times S)$ is defined,as usually, by

$$
a^{*}=\bigcup_{n=0}^{\infty} a^{n}
$$

where $a^{0}$ is the identity on $S$, and $a^{n}(n>0)$ is the composition $a ; a ; \ldots ; a, n$ times. The diamond operation $\langle a, p\rangle$ (for $a \in \exp (S \times S)$ and $p \in \exp (S))$ is defined to be the pre-image of $p$ under $a$,

$$
\left\{s \in S:\left(s, s^{\prime}\right) \in a, \text { for some } s^{\prime} \in p\right\} .
$$

Informally, the set $S$ can be interpreted as the set of states of a computer, the subsets of $S$ can be interpreted as propositions, the binary relations as computer programs. Then, $" s \in p$ " can be interpreted as "state $s$ satisfies proposition $p ",\left(s, s^{\prime}\right) \in a$ as "program a may run from initial state $s$ to final state $s$ ", $a ; b$ as "execute program $a$, then program $b$ ", $a \cup b$ as "execute program a or program $b$ non-deterministically", $a^{*}$ as "execute program a zero or more times", $\langle a, p\rangle$ as "the proposition satisfied when a is executed and stops in a state satisfying $p "$. Kripke structures are defined as the dynamic subalgebras of full Kripke structures. They intend to reflect the input-output behaviour of computer programs. Whenever we want to emphasize the fact that a certain Kripke structure $(\mathcal{B}, \mathcal{A})$ is a Kripke structure on a state set $S$ we will indicate it by $(\mathcal{B}, \mathcal{A}, S)$.

Full Kripke structures are separable dynamic algebras, though Kripke structures are not necessarily separable. Let us denote by SKri the full subcategory of $D A$ whose objects are the separable Kripke structures.

The simplest examples of dynamic algebras are the so called Boolean-trivial dynamic algebras $(\mathcal{B}, \mathcal{A},<>)$ with $\mathcal{B}=\{0=1\}, \mathcal{A}=(A, ;, \cup, *)$ of the required type and $\langle a, 0\rangle=0$. They are, obviously, non-separable.

Denote by FKri the class of all finite full Kripke structures and by $T$ the class of all Boolean-trivial dynamic algebras. Allow us to reuse the symbol $D A$ to denote the variety of dynamic algebras.

The importance of the above two types of examples was shown by Pratt [29] in the establishment of the Theorem,

$$
(*) D A=\operatorname{HSP}(F K r i \cup T),
$$


where $\mathrm{H}, \mathrm{S}$ and $\mathrm{P}$ stand for the closure under homomorphic images, subalgebras and products, respectively. Thus, $D A$ is the smallest variety containing FKri and determined by a set of Boolean equations only (i.e., equations on Boolean sort), namely, the set of Boolean equations satisfied in FKri . The equality $(*)$ is the algebraic counterpart of the Segerberg-ParikhGabbay completeness Theorem for PDL, which was established by means of the Segerberg's axioms (cf. [8], [28], [35]). Thus a Boolean equation is satisfied in every finite full Kripke structure iff it can be "deduced" from the equations (i)-(v) above. See, e.g. [33] and [13], to define a formal way to deduce new equations from old equations in such a way that Completeness Theorem can be established.

More about dynamic algebras and Kripke structures can be found in [17], [18], [20], [21], $[27],[31]$.

\subsection{Formulation of the Main Theorem}

We introduce perfect classes of Kripke structures. More details on those classes of Kripke structures will be given in section 2. Thus, a class $\mathcal{C}$ of Kripke structures is called perfect if, for every $(\mathcal{B}, \mathcal{A}, S),\left(\mathcal{B}^{\prime}, \mathcal{A}^{\prime}, S^{\prime}\right) \in \mathcal{C}$ and every homomorphism $\left(h_{1}, h_{2}\right):(\mathcal{B}, \mathcal{A}, S) \longrightarrow\left(\mathcal{B}^{\prime}, \mathcal{A}^{\prime}, S^{\prime}\right)$ there is a unique mapping $f: S^{\prime} \longrightarrow S$ such that $h_{1}(p)=f^{-1}(p)$ for every $p \in \mathcal{B}$ and there is no other $h_{2}^{\prime}: \mathcal{A} \longrightarrow \mathcal{A}^{\prime}$ such that $\left(f^{-1} / \mathcal{B}, h_{2}^{\prime}\right)$ is a homomorphism. The aim of this paper is to prove the following,

MAIN THEOREM. There exists a perfect class of separable Kripke structures such that the corresponding full subcategory of SKri is algebraically universal, which means that it contains any category of universal algebras as a full subcategory.

In section 2 of this paper we introduce state mappings, its relations with Kripke structure homomorphisms and define perfect class of Kripke structures. In section 3 we recall the representation problems concerning universality. In section 4 we refer an almost full embedding from which the main result will be proved and in sections 5 and 6 we prove it. In section 7 we list some of the consequences of the algebraic universality of $S K r i$. In section 8 we refer test algebras.

\section{State mappings}

Since Kripke structures are dynamic algebras, their homomorphisms are defined as dynamic algebra homomorphisms. However, homomorphisms of Kripke structures can carry more information. We define a state mapping $f:(\mathcal{B}, \mathcal{A}, S) \longleftarrow\left(\mathcal{B}^{\prime}, \mathcal{A}^{\prime}, S^{\prime}\right)$ as an arbitrary mapping $f: S^{\prime} \longrightarrow S$.

There is no relation between homomorphisms and state mappings in general, as we show below.

PROPOSITION 2.1. Let $f: S^{\prime} \longrightarrow S$ be a mapping. Then

$$
\left(f^{-1},(f \times f)^{-1}\right):(\exp (S), \exp (S \times S), S) \longrightarrow\left(\exp \left(S^{\prime}\right), \exp \left(S^{\prime} \times S^{\prime}\right), S^{\prime}\right)
$$

is a homomorphism iff $f$ is a bijection.

Obs.:Given a mapping $f: S^{\prime} \longrightarrow S$ and a subset $p \subseteq S$, we denote by $f^{-1}(p)$ the pre-image of $p$ under $f$.

Proof. If $f$ is a bijection, then $\left(f^{-1},(f \times f)^{-1}\right)$ is an isomorphism, evidently.

If $f$ is not a bijection, then either it is not one-to-one or it is not surjective. 
In $(\exp (S), \exp (S \times S), S)$ it happens $\emptyset^{*}=\Delta_{S}$. If $f$ is not one-to-one, then

$$
\left((f \times f)^{-1}(\emptyset)\right)^{*}=\emptyset^{*}=\Delta_{S^{\prime}} \neq(f \times f)^{-1}\left(\Delta_{S}\right) .
$$

If $f$ is not surjective, then the proposition just below can be used.

PROPOSITION 2.2. Let $f: S^{\prime} \rightarrow S$ be a mapping not surjective. Then there is no homomorphism

$$
\left(h_{1}, h_{2}\right):(\exp (S), \exp (S \times S), S) \rightarrow\left(\exp \left(S^{\prime}\right), \exp \left(S^{\prime} \times S^{\prime}\right), S^{\prime}\right)
$$

for which $h_{1}=f^{-1}$.

Proof. Let there exists $h_{2}: \exp (S \times S) \longrightarrow \exp \left(S^{\prime} \times S^{\prime}\right)$ such that $h=\left(f^{-1}, h_{2}\right)$ is a homomorphism. Since $f$ is not surjective, we can choose $s \in S \backslash f\left(S^{\prime}\right)$. Choose $a=\{s\} \times S$. Then $\langle a, S\rangle=\{s\}$, hence $\left\langle h_{2}(a), S^{\prime}\right\rangle=\emptyset$. So that $h_{2}(a)=\emptyset$. Choose $b=S \times\{s\}$. Then $<b a, S>=S$, hence $\left\langle h_{2}(b a), S^{\prime}>=S^{\prime}\right.$. But then $h_{2}(a b) \neq h_{2}(a) h_{2}(b)$.

State mappings can induce Kripke structure homomorphisms. Thus, we consider

DEFINITION 2.3. A state mapping

$$
f:(\mathcal{B}, \mathcal{A}, S) \longleftarrow\left(\mathcal{B}^{\prime}, \mathcal{A}^{\prime}, S^{\prime}\right)
$$

is compatible if there exists $h_{2}: \mathcal{A} \longrightarrow \mathcal{A}^{\prime}$ such that $h=\left(f^{-1} / \mathcal{B}, h_{2}\right)$ is a homomorphism. We say that $f$ is uniquely compatible if there exists precisely one such $h_{2}$.

EXAMPLE 2.4. Consider a state set $S$ with more than one element. Choose $s \in S$. Define the Kripke structure $(\mathcal{B}, \mathcal{A}, S)$ where $\mathcal{B}=\{\emptyset, S\}$ and $\mathcal{A}=\left\{\Delta_{S}, a, \Delta_{S} \cup a\right\}$ with $a=S \times\{s\}$. Then the identity on $S$ is a compatible, but not uniquely compatible state mapping $1_{S}:(\mathcal{B}, \mathcal{A}, S) \longleftarrow(\mathcal{B}, \mathcal{A}, S)$, since $\left(1_{S}^{-1} / \mathcal{B}, h_{2}^{\prime}\right)$ and $\left(1_{S}^{-1} / \mathcal{B}, h_{2}^{\prime \prime}\right)$ are homomorphisms where $h_{2}^{\prime}$ is the identity mapping on $\mathcal{A}$ and $h_{2}^{\prime \prime}$ is the constant mapping constantly equal to $\Delta_{S}$.

On the other hand Kripke structure homomrphisms can be induced by state mappings. So then, we consider

DEFINITION 2.5. A homomorphism

$$
\left(h_{1}, h_{2}\right):(\mathcal{B}, \mathcal{A}, S) \longrightarrow\left(\mathcal{B}^{\prime}, \mathcal{A}^{\prime}, S^{\prime}\right)
$$

is grounded if there exists a state mapping $f: S^{\prime} \longrightarrow S$ such that $h_{1}=f^{-1} / \mathcal{B}^{\text {. We say that }}$ $\left(h_{1}, h_{2}\right)$ is uniquely grounded if there exists precisely one state mapping $f$ with $h_{1}=f^{-1} / \mathcal{B}$.

EXAMPLE 2.6. Let $S$ be an infinite set. Consider the Kripke structure $(\mathcal{B}, \mathcal{A}, S)=$ $\left(\exp (S),\left\{\Delta_{S}\right\}, S\right)$. Let $\mathcal{U}$ be an ultrafilter on $\exp (S)$ satisfying $\{s\} \notin \mathcal{U}$ for every $s \in S$ (such an ultrafilter exists by Zorn's Lemma). Let $\left(\mathcal{B}^{\prime}, \mathcal{A}^{\prime}, S^{\prime}\right)$ be an arbitrary Kripke structure such that $\mathcal{A}^{\prime}$ contains $\Delta_{S^{\prime}}$. Then the homomorphism

$$
\left(h_{1}, h_{2}\right):(\mathcal{B}, \mathcal{A}, S) \longrightarrow\left(\mathcal{B}^{\prime}, \mathcal{A}^{\prime}, S^{\prime}\right)
$$

defined by

$$
h_{1}(p)=\left\{\begin{array}{lc}
S^{\prime} & \text { if } p \in \mathcal{U} \\
\emptyset & \text { otherwise }
\end{array}\right.
$$

and $h_{2}\left(\Delta_{S}\right)=\Delta_{S^{\prime}}$ is not grounded because $h_{1}$ doesn't preserve infinite unions. 
EXAMPLE 2.7. Consider the Kripke structure $(\mathcal{B}, \mathcal{A}, S)$ on $S=\left\{s_{1}, s_{2}\right\}$ with $\mathcal{B}=\{\emptyset, S\}$, $\mathcal{A}=\left\{\Delta_{S}\right\}$. Then, for every Kripke structure $\left(\mathcal{B}^{\prime}, \mathcal{A}^{\prime}, S^{\prime}\right)$ such that $\mathcal{A}^{\prime}$ contains $\Delta_{S^{\prime}}$, the homomorphism

$$
\left(h_{1}, h_{2}\right):(\mathcal{B}, \mathcal{A}, S) \longrightarrow\left(\mathcal{B}^{\prime}, \mathcal{A}^{\prime}, S^{\prime}\right)
$$

defined by $h_{1}(\emptyset)=\emptyset, h_{1}(S)=S^{\prime}$ and $h_{2}\left(\Delta_{S}\right)=\Delta_{S^{\prime}}$ is grounded but not uniquely grounded, since $h_{1}=f^{-1 / \mathcal{B}}=g^{-1 / \mathcal{B}}$ for $f, g: S^{\prime} \longrightarrow S$ state mappings defined by $f(s)=s_{1}, g(s)=s_{2}$ for $s \in S^{\prime}$.

Now, we can rewrite the definition of perfect class of Kripke structures.

DEFINITION 2.8. A class $\mathscr{C}$ of Kripke structures is called perfect if, for every $(\mathcal{B}, \mathcal{A}, S)$, $\left(\mathcal{B}^{\prime}, \mathcal{A}^{\prime}, S^{\prime}\right) \in \mathcal{C}$, every homomorphism $h:(\mathcal{B}, \mathcal{A}, S) \longrightarrow\left(\mathcal{B}^{\prime}, \mathcal{A}^{\prime}, S^{\prime}\right)$ is uniquely grounded and the corresponding state mapping is uniquely compatible.

\section{On algebraic universality}

\subsection{Representation problems}

The representation problem for groups mentioned in the abstract has its simplest form when we choose $(I, \leq)$ to be a singular set trivially ordered, that is, "Given a category $\mathcal{U}$, is every group isomorphic to the group of all automorphisms of some object of $\mathcal{U}$ ?". This is the case with the category of symmetric graphs, the category of distributive lattices, the category of topological spaces, (cf. [7], [34], [3], [9]).

A similar question can be formulated for monoids instead of groups as "Given a category $\mathcal{U}$, is every monoid $M$ isomorphic to the monoid of all endomorphisms of some object of $\mathcal{U}$ ?". That is, "Is there some object $A \in O b j \mathcal{U}$ such that $M \cong \mathcal{U}(A, A)$ ?". The category of binary relations, the category of semigroups, the category of topological spaces and open continuous mappings satisfy that condition.

Often, the answer to those questions requires a category-theoretical reasoning not only in the part where one represents but in the part which we represent, also. Since a monoid can be seen as a (only one object) category, we are led to a generalization from a problem of representation of monoids to a problem of representation of categories.

We are interested in the representation of the concretizable categories. Recalling the definition, we say that a category $\mathcal{C}$ is concretizable if there exists a faithful functor

$$
\mathcal{F}: \mathcal{C} \longrightarrow \text { Set }
$$

where Set is the category of all sets and all mappings. Now, our representation problem can be reformulated as follows "Given a category $\mathcal{U}$, can every concretizable category $\mathcal{C}$ be represented in $\mathcal{U}$ in such a way that objects of $\mathcal{C}$ are substituted by objects of $\mathcal{U}$ and the morphisms between every two objects of $\mathcal{C}$ get represented exactly by the morphisms prescribed in $\mathcal{U}$ between the corresponding image objects". In other words, "Is $\mathcal{C}$ isomorphic to a full subcategory of $\mathcal{U}$ ?". Whenever a category $\mathcal{U}$ has the above property it is called an universal category. An example of an universal category is the category $S\left(P^{+}\right)$whose objects are pairs $(X, \mathscr{S})$ where $X$ is a set and $\mathscr{S}$ is a family of subsets of $X$ and morphisms from $(X, \mathscr{S})$ to $\left(X^{\prime}, \mathscr{S}^{\prime}\right)$ are mappings $f: X \rightarrow X^{\prime}$, such that each $S$ belonging to $\mathscr{S}$ has image under $f, f(S)$, lying in $\mathscr{S}^{\prime}$.

A category is said to be algebraic if it is isomorphic to a category of some algebras of a given type and all their homomorphisms. A category is algebraically universal (briefly, alguniversal) if every algebraic category is fully embeddable into it. We recall that a category 
$\mathcal{A}$ is fully embeddable into a category $\mathcal{B}$ if there is a full one-to-one functor $F: \mathcal{A} \rightarrow \mathcal{B}$. The category $\operatorname{Alg}(\Delta)$ of all algebras of a given type $\Delta$ is alg-universal iff the sum of the arities of the operations of type $\Delta$ is not less than 2. The category Graph of all graphs is also alguniversal. Moreover, this category provides a criterion to decide about the alg-universality of any category, since

\section{A category is alg-universal iff the category Graph fully embeds into it.}

The above results are contributions of Kučera, Hedrlín, Pultr and Trnková. A full account of universality and alg-universality can be found in the Pultr and Trnková's monograph [30].

Under the set-theoretical assumption that there are not too many measurable cardinals, more precisely, that

(M) There exists a cardinal $\alpha$ such that every $\alpha$-additive two-valued measure is trivial,

every concretizable category is algebraic (cf. [10], [22]). Then, Kučera and Pultr concluded in [23] that concretizability coincides with algebraicity iff $(\mathrm{M})$ (e.g. the category of compact Hausdorff spaces and continuous mappings is non-algebraic under non (M)).

Clearly, under condition (M) both the concepts of universality and of algebraic universality coincide. Therefore, in this paper we restrict ourselves to the problem of the alg-universality of the category of separable Kripke structures. The category of dynamic algebras is, trivially, alg-universal since its full subcategory whose objects are the Boolean-trivial dynamic algebras is isomorphic to the category $\operatorname{Alg}(2,2,1)$. Moreover, the category of Boolean algebras is not alg-universal because $\mathbb{Z}_{3}$, the cyclic group of order 3 , is an automorphism group of no Boolean algebra [26]. This is of relevance to our problem in that the investigation of the

alg-universality of separable Kripke structures may now become one of choosing a class of Boolean algebras with a parallel interfering structure, the action part of the Kripke structure, which will determine the compatible Boolean homomorphisms.

\subsection{Remarks on the definition of algebraic category}

The concept of algebraic category was introduced by Isbell in [14], where homogeneous algebras, that is, algebras with one underlying set only, were understood. Actually, the algebraicity doesn't need to be described by (full) operations, since each $\operatorname{Rel}(\Delta)$, the category of all relational systems of type $\Delta$ and all their homomorphisms, is algebraic as shown by Hedrlín and Pultr in [11].

Here, we deal with dynamic algebras, which are heterogeneous algebras, that is, algebras with possibly many underlying sets ( $c f$. [4] and [2] for the formal definition). However, categories of heterogeneous algebras and all their homomorphisms are algebraic, too. It is enough to see each heterogeneous algebra $\mathcal{A}$ as relational system defined on the set-theoretical disjoint union of the underlying sets of $\mathcal{A}$ with a relation corresponding to each operation of A (the relation which is the operation seen as a relation) plus a unary relation per each, and equal to each, one of the underlying sets of $\mathcal{A}$. The unary relations are added to choose the right relation homomorphisms.

\section{Preliminary construction}

Let $U n d G r a p h_{0}$ denote the category of connected undirected graphs without loops with more than one vertex and all their homomorphisms. It is known (cf. [30]) that UndGraph 0 is alguniversal. Therefore, to prove the Main Theorem it is enough to construct a full embedding of $U n d G r a p h_{0}$ into SKri in such a way that the image subcategory defines a perfect class of Kripke structures. The desired full embedding will be a composition of two contravariant 
embeddings, say $\phi \circ \psi$. We will restrict the domain of $\phi$ to the image $\psi\left(U n d G r a p h_{0}^{o p}\right)$. So, we need to describe this category. This is will be the aim of this section (we follow the monograph [30]). The embedding $\phi$ is constructed in section 5. We preserve the notation used in this section in the forthcoming sections.

By Comp we denote the category of compact Hausdorff spaces and continuous mappings. Comp is not alg-universal, although its dual is almost alg-universal, that is the required embedding is full up to the constant morphisms, more exactly

THEOREM 4.1.([37],[30]). There exists an almost full embedding

$$
\psi: \operatorname{UndGraph}_{0}^{o p} \rightarrow \text { Comp }
$$

Thus, by definition, we have

(i) $\psi$ is one-to-one on objects and on morphisms;

(ii) For any morphism $f$ of $U n d G r a p h_{0}, \psi(f)$ is a non-constant continuous mapping;

(iii) For every pair of graphs $(X, R),\left(X^{\prime}, R^{\prime}\right)$ belonging to $U n d G r a p h_{0}$ and every nonconstant morphism $g: \psi\left(X^{\prime}, R^{\prime}\right) \rightarrow \psi(X, R)$ in Comp there exists a morphism $f:(X, R) \rightarrow\left(X^{\prime}, R^{\prime}\right)$ in $U_{n d G r a p h}$, such that $\psi(f)=g$.

In this case, Comp is said to be dual to an almost alg-universal category. In order to describe the category $\psi\left(U n d G r a p h^{o p}\right)$, we recall some definitions.

We define continua as connected compact Hausdorff spaces with more than one point. Such spaces are, consequently, infinite sets. A Cook continuum is a continuum $D$ such that for any subcontinuum $S$ of $D$, each continuous mapping $f: S \rightarrow D$ is either a constant mapping or the inclusion. Continua with this property were given the name Cook continuum, since it was H. Cook [6] who firstly constructed such a continuum. Each continuum has a countable pairwise disjoint system of its subcontinua. See the Appendix A of [30] for details.

We recall that a topological space $D$ is said rigid if each continuous mapping $f: D \rightarrow D$ is either a constant mapping or the identity. The following property is satisfied:

(1) Let $X, X^{\prime}$ be sets and $D$ a rigid space. Denote by $D^{X}$ the product space (i.e., with the topology of the pointwise convergence). Let $a$ and $b$ be distinct elements of $D$. Denote by $c_{a}$ $\left(\right.$ resp. $c_{a}^{\prime}$ ) the element of $D^{X}$ (resp. $D^{X^{\prime}}$ ) constantly equal to a. Consider, similarly, $c_{b}$ and $c_{b}^{\prime}$. Let $g: D^{X^{\prime}} \rightarrow D^{X}$ be a continuous mapping such that $g\left(c_{a}^{\prime}\right)=c_{a}$ and $g\left(c_{b}^{\prime}\right)=g\left(c_{b}\right)$. Then, there exists a mapping $f: X \rightarrow X^{\prime}$ such that $g(\alpha)=\alpha_{\circ} f$ for all $\alpha \in D^{X^{\prime}}$.

Given a graph $(X, R)$ we say that $Y \subseteq X$ is an independent set of $(X, R)$ if no two vertices of $Y$ are joined by an edge, i.e., if $\{x, y\} \subseteq Y \Longrightarrow\{x, y\} \notin R$. A characteristic mapping $h_{Y}: X \longrightarrow\{0,1\}$ of a set $Y \subseteq X$ is defined to be $h_{Y}(y)=1$ for $y \in Y$ and $h_{Y}(y)=0$ otherwise.

Now, we proceed with the construction of $\psi$, which is done after several steps, as follows:

- Choose $A, B, C, H$ pairwise disjoint subcontinua of a Cook continuum. Thus, between two distinct continua of the system $A, B, C, H$ no other continuous mappings are allowed than the constant ones. Moreover, each one of those spaces is rigid.

- Choose distinct elements $a_{0}, a_{1} \in A, b_{0}, b_{1} \in B, c_{0}, c_{1} \in C, 0,1, a, b \in H$.

- Given a graph $(X, R) \in U n d G r a p h_{0}$ a compact Hausdorff space $\psi(X, R)$ is constructed considering the following elements: 
- the product space $H^{X}$;

- $c_{a}$ and $c_{b}$ the elements of $H^{X}$ constantly equal, respectively, to $a$ and to $b$;

- the subspace $\chi_{R} \subseteq\{0,1\}^{X} \subseteq H^{X}$ consisting of the characteristic mappings of all independent sets of $(X, R)$. The set $\chi_{R}$ is a closed subset of $H^{X}$ ([30, VI.16.9]);

- the following identifications in the topological sum

$$
\begin{aligned}
& S=A \vee B \vee H^{X} \vee\left(\chi_{R} \times C\right) \\
a_{0} & \sim c_{a}, \\
b_{0} & \sim c_{b}, \\
h_{Y} & \sim\left(h_{Y}, c_{0}\right) \text { for all } h_{Y} \in \chi_{R}, \\
a_{1} & \sim b_{1} \sim\left(h_{Y}, c_{1}\right) \text { for all } h_{Y} \in \chi_{R} .
\end{aligned}
$$

- Define $\psi(X, R)$ as the quotient space $S / \sim$. Since $S$ is a finite sum of compact spaces, $S$ is still compact. Then, $\psi(X, R)$ is compact and it is easily seen that it is Hausdorff, too.

To simplify the notation, allow us to suppose that $A, B, H^{X}, \chi_{R} \times C$ are subsets of $\psi(X, R)$ and $a_{0}=c_{a}, \ldots, a_{1}=b_{1}=\left(h_{Y}, c_{1}\right)$.

Let $f:(X, R) \longrightarrow\left(X^{\prime}, R^{\prime}\right)$ be a morphism in UndGraph ${ }_{0}$,

$$
\psi(f)=g: \psi\left(X^{\prime}, R^{\prime}\right) \longrightarrow \psi(X, R)
$$

is defined as follows,

$$
\begin{aligned}
& g(z)=z \text { for } z \in A \cup B, \\
& g(\alpha)=\alpha \circ f \text { for } \alpha \in H^{X^{\prime}}, \\
& g\left(h_{Y}, z\right)=\left(h_{Y} \circ f, z\right) \text { for }\left(h_{Y}, z\right) \in \chi_{R^{\prime}} \times C .
\end{aligned}
$$

The mapping $g$ is correctly defined, since it preserves the identifications made in the definition of the space $\psi(X, R)$. It is straightforward that $g$ is continuous. Clearly, $\psi$ is a one-to-one functor. A detailed proof of the fullness can be found in [30, VI.16.8-VI.16.14]. For the sake of clarity, we present a sketch of that proof.

Let

$$
g: \psi\left(X^{\prime}, R^{\prime}\right) \longrightarrow \psi(X, R)
$$

be a non-constant continuous mapping. According to the following properties:

(2) Let $L$ be one of the spaces $H, A, B, C$. Let $d: L \longrightarrow \psi(X, R)$ be a non-constant continuous mapping. Then, either $L \in\{A, B\}$ and $d(z)=z$ for all $z \in L$, or $L=C$ and there exists precisely one independent set $Y$ such that $d(z)=\left(h_{Y}, z\right)$ for all $z \in L$, or $L=H$ and $g(L) \subseteq H^{X}$

(3) Let $d: H^{X^{\prime}} \rightarrow \psi(X, R)$ be a non-constant continuous mapping. Then $d\left(H^{X^{\prime}}\right) \subseteq H^{X}$;

the mapping $g$ cannot be constant neither on $A, B, H^{X^{\prime}}$ nor $\left\{h_{Y}\right\} \times C$ for no $h_{Y} \in \chi_{R^{\prime}}$. Thus, by (2) and (3) again $g\left(H^{X^{\prime}}\right) \subseteq H^{X}$ and $g(z)=z$ for all $z \in A \cup B$. Therefore $g\left(c_{a}^{\prime}\right)=c_{a}$ and $g\left(c_{b}^{\prime}\right)=c_{b}$. By property (1) there exists a mapping $f: X \rightarrow X^{\prime}$ such that $g(\alpha)=\alpha \circ f$ for all $\alpha \in H^{X^{\prime}}$. Then, $g\left(h_{Y}, c_{1}\right)=\left(h_{Y} \circ f, c_{1}\right)$ for every $h_{Y} \in \chi_{R^{\prime}}$, which means that $f$ is a homomorphism of undirected graphs. Again, by property (2) we have $g\left(h_{Y}, z\right)=\left(h_{Y \circ} f, z\right)$ for all $h_{Y} \in \chi_{R^{\prime}}, z \in C$. We conclude $\psi(f)=g$.

The next Lemma is for later use. It will help us to calculate the interiors and the closures of subsets of the spaces of the form $\psi(X, R)$ for some graph $(X, R)$. 
Before proceeding we will fix some notation. By use of "cl", "int", "ext" and "b" we denote, respectively, the closure, the interior, the exterior and the boundary taken in the space which will index those keywords. Whenever the index space is $Q$, we won't use the index if there is no ambiguity. We also we use the bar - to denote the closure of subsets of $Q$. Given a set $S$ we denote by $S^{c}$ the complement of $S$. We denote the canonical projection by $p: S \longrightarrow S / \sim=\psi(X, R)$.

LEMMA 4.2. Let $G$ be a subset of $\psi(X, R)$. Then

(i) $\bar{G}=p\left(c l_{S}\left(p^{-1}(G)\right)\right)$;

(ii) $p\left(\operatorname{int}_{S}\left(p^{-1}(G)\right)\right)$ is open $\Longrightarrow \operatorname{int}(G)=p\left(\operatorname{int}_{S}\left(p^{-1}(G)\right)\right)$.

Proof. (i) Firstly, consider $F$ closed in $S$. Then $F$ is compact. Therefore, $p(F)$ is compact in the Hausdorff space $\psi(X, R)$. That is, $p(F)$ is closed. The immediate consequence is that $p\left(c l_{S}\left(p^{-1}(G)\right)\right.$ is closed. Now, the question is whether $p\left(c l_{S}\left(p^{-1}(G)\right)\right.$ is the least closed set in $\psi(X, R)$ containing $G$, which is obvious.

(ii) Mutatis mutandis. Note that $p\left(\operatorname{int}_{S}\left(p^{-1}(G)\right)\right)$ is not open in general. Choose, for instance, $G=A$.

\section{Construction of the embedding}

$$
\phi: \boldsymbol{\psi}\left(\operatorname{UndGraph} \mathbf{0}^{\text {op }}\right) \longrightarrow S K r i{ }^{\text {op }}
$$

The construction of $\phi$ is made after two steps. Several lemmas are established to insure the correct definition of $\phi$. Let $(X, R)$ be a graph of $U n d G r a p h_{0}$. Let's denote $Q=\psi(X, R)$. In order to define $\phi(Q)$ we are interested in a family of subsets of $Q$ stable under pre-images under non-constant continuous mappings. For that we will choose a particular family $\mathcal{T}$ of regularly open subsets of $Q$ and prove that it is a basis of $Q$. We recall that an open subset is said regularly open (r.o. for brevity) if it is equal to the interior of its closure. The basis $\mathcal{T}$ will determine the Boolean part and also the action part of $\phi(Q)$ and was refined in a way that, concerning the required fullness for $\phi$, the Boolean part will contribute to the definition of a convenient mapping and the action part will force the continuity of that mapping. We choose $y_{1}, y_{2}$ distinct points of $A \backslash\left\{a_{0}, a_{1}\right\}$ to be added to the structure of $\phi(Q)$ in a manner that the continuous mapping defined won't be constant.

We divide the construction of $\mathcal{T}$ into 8 parts to describe the specific situation around the points $a_{0}, a_{1}, \ldots, h_{Y}$ (for $h_{Y} \in \chi_{R}$ ) in $Q$. That is, $\mathcal{T}$ is defined to be the union

$$
\mathcal{T}=\mathcal{T}_{1} \cup \mathcal{T}_{2} \cup \cdots \cup \mathcal{T}_{8},
$$

where

$\mathcal{T}_{1}=\left\{U_{A} \subseteq Q: U_{A}\right.$ r.o. of $\left.A, a_{0}, a_{1} \notin c l_{A}\left(U_{A}\right), y_{1}, y_{2} \notin b_{A}\left(U_{A}\right)\right\}$,

$\mathcal{T}_{2}=\left\{U_{B} \subseteq Q: U_{B}\right.$ r.o. of $\left.B, b_{0}, b_{1} \notin c l_{B}\left(U_{B}\right)\right\}$,

$\mathcal{T}_{3}=\left\{U_{H^{X}} \subseteq Q: U_{H^{X}} \in \mathcal{T}_{0}, c_{a}, c_{b} \notin c l_{H^{X}}\left(U_{H^{X}}\right)\right.$ and $\left.c l_{H^{X}}\left(U_{H^{X}}\right) \cap \chi_{R}=\emptyset\right\}$,

here, $\mathcal{T}_{0}$ denotes the set

$\left\{\prod_{x \in X} U_{x}: U_{x}\right.$ r.o. of $H, x \in X$ and only a finite number of $U_{x}$ 's is different of $\left.H\right\}$ 


$$
\begin{aligned}
& \mathcal{T}_{4}=\left\{\left(U_{H^{x}} \cap \chi_{R}\right) \times U_{C} \subseteq Q: U_{H^{x}} \in \mathcal{T}_{0}, U_{C} \text { is a r.o. of } C, c_{0}, c_{1} \notin c l_{C}\left(U_{C}\right)\right\} \text {, } \\
& \mathcal{T}_{5}=\left\{U_{A} \bigcup U_{H^{X}} \subseteq Q: U_{A} \text { r.o. of } A, a_{0} \in U_{A}, a_{1} \notin c l_{A}\left(U_{A}\right), y_{1}, y_{2} \notin b_{A}\left(U_{A}\right)\right. \\
& \left.U_{H^{X}} \in \mathcal{T}_{0}, c_{a} \in U_{H^{X}}, c_{b} \notin c l_{H^{X}}\left(U_{H^{X}}\right), c l_{H^{X}}\left(U_{H^{X}}\right) \cap \chi_{R}=\emptyset\right\}, \\
& \mathcal{T}_{6}=\left\{U_{B} \cup U_{H^{X}} \subseteq Q: U_{B} \text { r.o. of } B, b_{0} \in U_{B}, b_{1} \notin c l_{B}\left(U_{B}\right)\right. \text {, } \\
& \left.U_{H^{X}} \in \mathcal{T}_{0}, c_{b} \in U_{H^{X}}, c_{a} \notin c l_{H^{X}}\left(U_{H^{X}}\right), c l_{H^{X}}\left(U_{H^{X}}\right) \cap \chi_{R}=\emptyset\right\}, \\
& \mathcal{T}_{7}=\left\{U_{H^{X}} \bigcup\left(U_{H^{X}} \cap \chi_{R}\right) \times U_{C} \subseteq Q: U_{H^{X}} \in \mathcal{T}_{0}, c_{a}, c_{b} \notin c l_{H^{X}}\left(U_{H^{X}}\right),\right. \\
& \left.c l_{H^{x}}\left(U_{H^{x}}\right) \cap \chi_{R}=U_{H^{x}} \cap \chi_{R} \neq \emptyset, U_{C} \text { r.o. of } C, c_{0} \in U_{C}, c_{1} \notin c l_{C}\left(U_{C}\right)\right\} \text {, } \\
& \mathcal{T}_{8}=\left\{U_{A} \bigcup U_{B} \bigcup\left(\chi_{R} \times U_{C}\right) \subseteq Q: U_{A} \text { r.o. of } A, a_{1} \in U_{A}, a_{0} \notin c l_{A}\left(U_{A}\right)\right. \text {, } \\
& y_{1}, y_{2} \notin b_{A}\left(U_{A}\right), U_{B} \text { r.o. of } B, b_{1} \in U_{B}, b_{0} \notin c l_{B}\left(U_{B}\right), U_{C} \text { r.o. of } C, c_{1} \in U_{C} \text {, } \\
& \left.c_{0} \notin c l_{C}\left(U_{C}\right)\right\} \text {. }
\end{aligned}
$$

LEMMA 5.1. The family $\mathcal{T}$ is a basis for the topological space $Q$.

Proof. Let $G$ be an open in $Q$, that is, $p^{-1}(G)$ is an open in $S$, where $p$ is the canonical projection. Consider $z \in G$. We are going to show that there exists $U \in \mathcal{T}$ such that $z \in U \subset G$.

The spaces $A, B, C, H$ are regular, since they are compact Hausdorff spaces. Thus, each one of them has a basis of regularly open sets.

We have several cases for $z$ :

1. $z \in A \backslash\left\{a_{0}, a_{1}\right\}$.

Therefore there exists a regularly open $U_{A}$ in $A$ such that

$$
z \in U_{A} \subseteq p^{-1}(G) \cap A .
$$

$A$ is Hausdorff and regular, so it is possible to choose $U_{A}$ such that $a_{0}, a_{1} \notin c l_{A}\left(U_{A}\right)$ and $y_{1}, y_{2} \notin b_{A}\left(U_{A}\right)$. Thus, $U_{A}=p^{-1}\left(U_{A}\right)$ is an open in $Q$ belonging to $\mathcal{T}_{1}$.

2. $z \in B \backslash\left\{b_{0}, b_{1}\right\}$.

Mutatis mutandis for 1 .

3. $z \in H^{X} \backslash\left(\left\{c_{a}, c_{b}\right\} \bigcup \chi_{R}\right)$.

Therefore there exists $\prod_{x \in X} U_{x} \in \mathcal{T}_{0}$, such that

$$
z \in \prod_{x \in X} U_{x} \subseteq p^{-1}(G) \cap H^{X} .
$$

Since $\chi_{R}$ is closed in $H^{X}$ (cf. 4) and since $H^{X}$ is Hausdorff and regular, we can choose $\prod_{x \in X} U_{x}$ such that

$$
c l_{H^{X}}\left(\prod_{x \in X} U_{x}\right) \cap\left(\chi_{R} \cup\left\{c_{a}, c_{b}\right\}\right)=\emptyset .
$$

Moreover $\prod_{x \in X} U_{x}$ is open in $Q$ since $p^{-1}\left(\prod_{x \in X} U_{x}\right)=\prod_{x \in X} U_{x}$. Thus, $\prod_{x \in X} U_{x} \in \mathcal{T}_{3}$.

4. $z \in \chi_{R} \times\left(C \backslash\left\{c_{0}, c_{1}\right\}\right)$.

Mutatis mutandis for 1 . 
5. $z=a_{0}=c_{a}$.

On one hand, there exists $U_{A}$ regularly open in $A$ such that

$$
a_{0} \in U_{A} \subseteq p^{-1}(G) \cap A .
$$

Again, since $A$ is by Hausdorff and regular it is possible to choose $U_{A}$ such that $a_{1} \notin c_{A}\left(U_{A}\right)$ and $y_{1}, y_{2} \notin b_{A}\left(U_{A}\right)$. On the other hand, there exists $U_{H^{X}} \in \mathcal{T}_{0}$ such that

$$
c_{a} \in U_{H^{X}} \subseteq p^{-1}(G) \cap H^{X} .
$$

And since $H^{X}$ is Hausdorff and regular it is possible to choose $U_{H^{X}}$ such that

$$
c l_{H^{X}}\left(U_{H^{X}}\right) \cap\left(\chi_{R} \cup\left\{c_{b}\right\}\right)=\emptyset .
$$

Therefore $U=U_{A} \cup U_{H^{X}}$ is an open in $Q$ belonging to $\mathcal{T}_{5}$.

6. $z=b_{0}=c_{b}$.

Mutatis mutandis for 5.

7. $z=h_{Y}=\left(h_{Y}, c_{0}\right) \in \chi_{R}$.

In this case there exists $U_{1} \in \mathcal{T}_{0}$ such that

$$
h_{Y} \in U_{1} \subseteq p^{-1}(G) \cap H^{X} .
$$

We can choose $U_{1}$ in such a way that

$$
c_{a}, c_{b} \notin c l_{H^{X}}\left(U_{1}\right) .
$$

Concurrently, there are $U_{2} \in \mathcal{T}_{0}$ and $U_{C}$ regularly open in $C$, such that

$$
\left(h_{Y}, c_{0}\right) \in\left(U_{2} \cap \chi_{R}\right) \times U_{C} \subseteq p^{-1}(G) \cap\left(\chi_{R} \times C\right) .
$$

$U_{C}$ can be chosen such that

$$
c_{1} \notin c l_{C}\left(U_{C}\right) .
$$

Let's put $U^{\prime}=U_{1} \cap U_{2}$. We can choose $\prod_{x \in X} U_{x} \in \mathcal{T}_{0}$, such that $\prod_{x \in X} U_{x} \subseteq U^{\prime}, h_{Y}(x) \in U_{x}$ and $c l_{H}\left(U_{x}\right) \bigcap\{0,1\}=U_{x} \bigcap\{0,1\}$ for each $x \in X$. Thus, $U=\left(\prod_{x \in X} U_{x}\right) \cup\left(\left(\prod_{x \in X} U_{x}\right) \cap \chi_{R}\right) \times U_{C}$ is an open in $Q$ belonging to $\mathcal{T}_{7}$.

8. $z=a_{1}=b_{1}=\left(h_{Y}, c_{1}\right), \forall h_{Y} \in \chi_{R}$.

Therefore, there are regularly open sets $U_{A}, U_{B}, U_{C}$ in each space such that

$$
z \in U_{A} \cup U_{B} \cup\left(\chi_{R} \times U_{C}\right) \subseteq p^{-1}(G) \cap\left(A \cup B \cup\left(\chi_{R} \times C\right)\right)
$$

Moreover, $U_{A}, U_{B}, U_{C}$ can be chosen such that $a_{0} \notin c l_{A}\left(U_{A}\right), y_{1}, y_{2} \notin b_{A}\left(U_{A}\right)$, $b_{0} \notin c l_{B}\left(U_{B}\right), c_{0} \notin c l_{C}\left(U_{C}\right)$. The open set $U_{A} \bigcup U_{B} \bigcup\left(\chi_{R} \times U_{C}\right)$ belongs to $\mathcal{T}_{8}$. 
STEP 1 . We define $\phi(Q)=\left(\mathcal{B}_{Q}, \mathcal{A}_{Q}\right)$, where

- $\mathcal{B}_{Q}$ is the Boolean subalgebra of $\exp (Q)$ generated by $\mathcal{T} \cup\left\{\left\{y_{1}\right\},\left\{y_{2}\right\}\right\}$;

- $\mathcal{A}_{Q}$ is the subalgebra of $\exp (Q \times Q)$ relative to the operations $\cup$, 。 and $*$ generated by $\left\{\Delta_{U}: U \in \mathcal{T}\right\} \cup\left\{Q \times\left\{y_{1}\right\}, Q \times\left\{y_{2}\right\}\right\}$ for $\Delta_{U}=\{(x, x): x \in U\}$.

LEMMA 5.2. $\mathcal{A}_{Q}$ is the set of binary relations on $Q$ of the following type

$$
\Delta_{G} \cup G_{1} \times\left\{y_{1}\right\} \cup G_{2} \times\left\{y_{2}\right\}
$$

for every $G, G_{1}, G_{2}$ belonging to the subalgebra $\mathcal{B}_{\mathcal{T}}$ of $(\exp (Q), \cup, \cap)$ generated by $\mathcal{T}$.

The proof is an easy exercise in the Kripke structure operations. Notice that the elements of $\mathcal{B}_{\mathcal{T}}$ are open sets in $Q$.

LEMMA 5.3. $\left(\mathcal{B}_{Q}, \mathcal{A}_{Q}\right)$ is a separable Kripke structure.

Proof. We have to show the closure under $<>$. Let $p \in \mathcal{B}_{Q}$. It is enough to prove the closure for $\Delta_{G}, G \times\{y\} \in \mathcal{A}_{Q}$ :

- $<\Delta_{G}, p>=G \cap p \in \mathcal{B}_{Q}$,

- $<G \times\{y\}, p>=\left\{\begin{array}{ll}G & \text { if } y \in p \\ \emptyset & \text { otherwise }\end{array} \in \mathcal{B}_{Q}\right.$.

It remains to prove that $\left(\mathcal{B}_{Q}, \mathcal{A}_{Q}\right)$ is separable. Let $R_{1}, R_{2}$ be binary relations belonging to $\mathcal{A}_{Q}$, such that $R_{1} \neq R_{2}$. Suppose $(x, y) \in R_{1} \backslash R_{2}$.

- If $y \in\left\{y_{1}, y_{2}\right\}$ then $\{y\} \in \mathcal{B}_{Q}$. We have $x \in<R_{1},\{y\}>$, but $x \notin<R_{2},\{y\}>$.

- If $y \notin\left\{y_{1}, y_{2}\right\}$ then there exists $\Delta_{G} \subseteq R_{1}$, such that $y \in G$ and $y=x$. Hence, $x \in<R_{1}, G \backslash\left\{y_{1}, y_{2}\right\}>$. Suppose that $x \in<R_{2}, G \backslash\left\{y_{1}, y_{2}\right\}>$. Then, there exists $y^{\prime} \in$ $G \backslash\left\{y_{1}, y_{2}\right\}$, such that, $\left(x, y^{\prime}\right) \in R_{2}$. Furthermore $y^{\prime} \neq y$, i.e., $y^{\prime} \neq x$. Therefore $y^{\prime} \in\left\{y_{1}, y_{2}\right\}$, which is a contradiction.

STEP 2. Let $\left(X^{\prime}, R^{\prime}\right)$ be another graph in UndGraph 0 . Let $Q^{\prime}, \mathcal{T}_{0}^{\prime}, \mathcal{T}_{1}^{\prime}, \ldots, \mathcal{T}_{8}^{\prime}, \mathcal{T}^{\prime}, c_{a}^{\prime}, c_{b}^{\prime}$ stand for $\left(X^{\prime}, R^{\prime}\right)$ as $Q, \mathcal{T}_{0}, \mathcal{T}_{1}, \ldots, \mathcal{T}_{8}, \mathcal{T}, c_{a}, c_{b}$ stand for $(X, R)$. If $g: Q^{\prime} \longrightarrow Q$ is a morphism of $\psi\left(\operatorname{UndGraph} h_{0}^{o p}\right)$, we define

$$
\phi(g)=\left(\phi_{g}^{1}, \phi_{g}^{2}\right):\left(\mathcal{B}_{Q}, \mathcal{A}_{Q}\right) \longrightarrow\left(\mathcal{B}_{Q^{\prime}}, \mathcal{A}_{Q^{\prime}}\right)
$$

by putting

(i) $\phi_{g}^{1}(p)=g^{-1}(p)$ for $p \in \mathcal{B}_{Q}$;

(ii) $\phi_{g}^{2}\left(\Delta_{G}\right)=\Delta_{g^{-1}(G)}$ for $\Delta_{G} \in \mathcal{A}_{Q}$;

(iii) $\phi_{g}^{2}(G \times\{y\})=g^{-1}(G) \times\{y\}$ for $G \times\{y\} \in \mathcal{A}_{Q}$;

(iv) For the remaining elements of $\mathcal{A}_{Q}$ use (ii), (iii) and the fact that $\phi_{g}^{2}$ preserves finite unions. 
REMARK 5.4. Suppose that $\phi$ is a well defined full embedding. Then all the homomorphisms $\left(h_{1}, h_{2}\right):\left(\mathcal{B}_{Q}, \mathcal{A}_{Q}\right) \longrightarrow\left(\mathcal{B}_{Q^{\prime}}, \mathcal{A}_{Q^{\prime}}\right)$ among the Kripke structures of the image subcategory of $\phi$ are of the described form, that is $\left(h_{1}, h_{2}\right)=\phi(g)$ for some continuous state mapping $g: Q^{\prime} \longrightarrow Q$. In particular, $h_{1}(p)=g^{-1}(p)$ for each $p$ belonging to the basis $\mathcal{T}$. Besides, for every $x \in Q$ the singular set $\{x\}$ is the intersection of all its open neighbourhoods belonging to $\mathcal{T}$ and pre-images under $g$ preserve those intersections. Therefore $\left(h_{1}, h_{2}\right)$ is uniquely grounded and $g$ is uniquely compatible. Thus, the mentioned class of Kripke structures will be perfect.

Since $\psi$ is a full embedding, we have $g=\psi(f)$ for one and only one morphism of graphs $f:(X, R) \longrightarrow\left(X^{\prime}, R^{\prime}\right)$.

The rest of this section is devoted to the proof that $\phi$ is a well defined embedding.

\section{LEMMA 5.5. We have}

(i) $g^{-1}\left(\left\{y_{1}\right\}\right)=\left\{y_{1}\right\} \in \mathcal{B}_{Q^{\prime}} \quad$ and $\quad g^{-1}\left(\left\{y_{2}\right\}\right)=\left\{y_{2}\right\} \in \mathcal{B}_{Q^{\prime}}$;

(ii) For $U \in \mathcal{T}, g^{-1}(U) \in \mathcal{T}^{\prime}$;

(iii) For $p \in \mathcal{B}_{Q}, g^{-1}(p) \in \mathcal{B}_{Q^{\prime}}$.

Proof. (i) Follows from last remark.

(iii) Follows from (i) and (ii).

(ii) Beginning with some remarks we will reach the aimed conclusions for each set $U \in \mathcal{T}$.

(a) For $\prod_{x \in X} U_{x} \in \mathcal{T}_{0}, g^{-1}\left(\prod_{x \in X} U_{x}\right)=\prod_{x^{\prime} \in X^{\prime}} U_{x^{\prime}}^{\prime} \in \mathcal{T}_{0}^{\prime}$ where $U_{x^{\prime}}^{\prime}=\bigcap_{x \in f^{-1}\left(\left\{x^{\prime}\right\}\right)} U_{x}$, as we are going to verify.

It happens that $g^{-1}\left(\prod_{x \in X} U_{x}\right) \subseteq g^{-1}\left(H^{X}\right) \subseteq H^{X^{\prime}}$. Then,

$\alpha \in g^{-1}\left(\prod_{x \in X} U_{x}\right) \Longleftrightarrow \alpha \circ f \in \prod_{x \in X} U_{x} \Longleftrightarrow \forall x \in X, \alpha(f(x)) \in U_{x} \Longleftrightarrow$

$$
\Longleftrightarrow \forall x^{\prime} \in X^{\prime}, \alpha\left(x^{\prime}\right) \in \bigcap_{x \in\left\{x: f(x)=x^{\prime}\right\}} U_{x}
$$

Notice that it may happen $\left\{x: x^{\prime}=f(x)\right\}=\emptyset$. In that case, we make the usual convention $\bigcap_{x \in \emptyset} U_{x}=H$. Moreover, each $U_{x}$ appears in the expression of only one of the $U_{x^{\prime}}^{\prime}$ otherwise the mapping $f$ wouldn't be a well defined mapping. Consequently, only finitely many $U_{x}$ 's are distinct from $H$ in the expression of $U_{x^{\prime}}^{\prime}$, then $U_{x^{\prime}}^{\prime}$ is a regularly open set. Therefore, we can conclude that the product $\prod_{x^{\prime} \in X^{\prime}} U_{x^{\prime}}^{\prime}$ is in $\mathcal{T}_{0}^{\prime}$.

(b) For $U \in \mathcal{T}_{0}$, we have

$$
c l_{H^{X^{\prime}}}\left(g^{-1}(U)\right) \subseteq g^{-1}\left(c l_{H^{x}}(U)\right) .
$$

Now, we consider the different types of elements of $\mathcal{T}$.

1. If $U \in \mathcal{T}_{1}$, trivially we have $g^{-1}(U)=U \in \mathcal{T}_{1}^{\prime}$.

2. If $U \in \mathcal{T}_{2}$, mutatis mutandis for 1 .

3. If $U \in \mathcal{T}_{3}$, it follows from the above remarks that $g^{-1}(U) \in \mathcal{T}_{3}^{\prime}$. 
4. If $U=\left(\left(\prod_{x \in X} U_{x}\right) \cap \chi_{R}\right) \times U_{C} \in \mathcal{T}_{4}$, we have

$$
g^{-1}\left(\left(\left(\prod_{x \in X} U_{x}\right) \cap \chi_{R}\right) \times U_{C}\right) \subseteq g^{-1}\left(\chi_{R} \times U_{C}\right) \subseteq \chi_{R^{\prime}} \times C .
$$

As a consequence, we can write

$$
g^{-1}\left(\left(\left(\prod_{x \in X} U_{x}\right) \cap \chi_{R}\right) \times U_{C}\right)=\left(\left(\prod_{x^{\prime} \in X^{\prime}} U_{x^{\prime}}^{\prime}\right) \cap \chi_{R}\right) \times U_{C} \in \mathcal{T}_{4}^{\prime} .
$$

5. If $U=U_{A} \bigcup \prod_{x \in X} U_{x} \in \mathcal{T}_{5}$, it happens that

$$
g^{-1}\left(U_{A} \cup\left(\prod_{x \in X} U_{x}\right)\right)=U_{A} \cup\left(\prod_{x^{\prime} \in X^{\prime}} U_{x^{\prime}}^{\prime}\right)
$$

Moreover, if $c_{a} \in\left(\prod_{x \in X} U_{x}\right)$ we have $c_{a}^{\prime} \in \prod_{x^{\prime} \in X^{\prime}} U_{x^{\prime}}^{\prime}$. Therefore, the conclusion that $g^{-1}(U) \in \mathcal{T}_{5}^{\prime}$ follows easily from the above remarks.

6. If $U \in \mathcal{T}_{6}$, mutatis mutandis for 5 .

7. If $U=\left(\prod_{x \in X} U_{x}\right) \cup\left(\left(\prod_{x \in X} U_{x}\right) \cap \chi_{R}\right) \times U_{C} \in \mathcal{T}_{7}$, we will have $g^{-1}(U) \in \mathcal{T}_{7}^{\prime} \cup \mathcal{T}_{3}^{\prime}$. In fact,

$$
\begin{gathered}
g^{-1}\left(\left(\prod_{x \in X} U_{x}\right) \cup\left(\left(\prod_{x \in X} U_{x}\right) \cap \chi_{R}\right) \times U_{C}\right)= \\
=\left(\prod_{x^{\prime} \in X^{\prime}} U_{x^{\prime}}^{\prime}\right) \cup\left(\left(\prod_{x^{\prime} \in X^{\prime}} U_{x^{\prime}}^{\prime}\right) \cap \chi_{R^{\prime}}\right) \times U_{C} .
\end{gathered}
$$

By remark (b), if a characteristic function $h_{Y} \in c l_{H^{X^{\prime}}}\left(\prod_{x^{\prime} \in X^{\prime}} U_{x^{\prime}}^{\prime}\right)$ then $g\left(h_{Y}\right)=h_{f^{-1}(Y)} \in c l_{H^{X}}\left(\prod_{x \in X} U_{x}\right)$. Since $U \in \mathcal{T}_{7}$, we have $g\left(h_{Y}\right) \in \prod_{x \in X} U_{x}$. Consequently, $h_{Y} \in \prod_{x^{\prime} \in X^{\prime}} U_{x^{\prime}}^{\prime}$. Therefore,

$$
c l_{H^{X^{\prime}}}\left(\prod_{x^{\prime} \in X^{\prime}} U_{x^{\prime}}^{\prime}\right) \cap \chi_{R^{\prime}}=\left(\prod_{x^{\prime} \in X^{\prime}} U_{x^{\prime}}^{\prime}\right) \cap \chi_{R^{\prime}}
$$

The conditions on $c_{a}^{\prime}$ and $c_{b}^{\prime}$ are also verified. Finally, if

$$
\left(\prod_{x^{\prime} \in X^{\prime}} U_{x^{\prime}}^{\prime}\right) \cap \chi_{R^{\prime}} \neq \emptyset
$$

then $g^{-1}(U) \in \mathcal{T}_{7}^{\prime}$. Otherwise, $g^{-1}(U) \in \mathcal{T}_{3}^{\prime}$.

8. If $U=U_{A} \cup U_{B} \bigcup\left(\chi_{R} \times U_{C}\right) \in \mathcal{T}_{8}$ then $g^{-1}(U)=U_{A} \bigcup U_{B} \bigcup\left(\chi_{R^{\prime}} \times U_{C}\right) \in \mathcal{T}_{8}^{\prime}$.

LEMMA 5.6. $\phi(g)$ is a well defined homomorphism of Kripke structures. 
Proof. It follows from Lemma 5.5 that $\phi_{g}^{1}$ is a well defined map. Besides, $\phi_{g}^{2}$ is a well defined mapping too, since each $R \in \mathcal{A}_{Q}$ admits only one decomposition $R=\Delta_{G} \cup G_{1} \times\left\{y_{1}\right\} \cup G_{2} \times\left\{y_{2}\right\}$ (is a consequence of the choice of the elements of $\mathcal{T}_{1}$, $\mathcal{T}_{5}$ and $\mathcal{T}_{8}$ ). $\phi_{g}^{1}$ is, trivially, a homomorphism of Boolean algebras. $\phi_{g}^{2}$ is additive by construction. The preservation of the composition is a direct consequence of the following fact

$\left.{ }^{*}\right)$ for $p \in \mathcal{B}_{Q}$ and $y \in\left\{y_{1}, y_{2}\right\}, \quad y \in p$ iff $y \in g^{-1}(p)$.

We will verify that $\phi_{g}^{2}$ preserves $*$. For $\Delta_{G} \cup G_{1} \times\left\{y_{1}\right\} \cup G_{2} \times\left\{y_{2}\right\} \in \mathcal{A}_{Q}$ we have

$$
\begin{aligned}
& \phi_{g}^{2}\left(\left(\Delta_{G} \cup G_{1} \times\left\{y_{1}\right\} \cup G_{2} \times\left\{y_{2}\right\}\right)^{*}\right)= \\
= & \Delta_{Q^{\prime}} \cup g^{-1}\left(G_{1}\right) \times\left\{y_{1}\right\} \cup g^{-1}\left(G_{2}\right) \times\left\{y_{2}\right\} \cup\left(Q^{\prime} \times \delta_{y_{1}}^{g^{-1}\left(G_{2}\right)} \cap g^{-1}\left(G_{1}\right) \times\left\{y_{2}\right\}\right) \cup \\
= & \left(\Delta_{Q^{\prime}} \cup g^{-1}\left(G_{1}\right) \times\left\{y_{1}\right\} \cup g^{-1}\left(G_{2}\right) \times\left\{y_{2}\right\}\right)^{*}= \\
= & \left.\left(\phi_{g}^{2}\left(\Delta_{G} \cup G_{1} \times\left\{y_{1}\right\} \cup G_{2} g_{2}^{-1}\left(G_{1}\right) \cap g_{2}\right\}\right)\right)^{*},
\end{aligned}
$$

where the Kronecker symbol $\delta$ is used with the following meaning, for $y \in Q^{\prime}$ and $F \subseteq Q^{\prime}$ :

$$
\delta_{y}^{F}=\left\{\begin{array}{l}
Q^{\prime} \text { if } y \in F \\
\emptyset \text { otherwise }
\end{array}\right.
$$

It remains to prove that $\phi_{g}$ preserves $<>$. It is enough to analyze the cases $\Delta_{G}, G \times\{y\} \in \mathcal{A}_{Q}$, for $p \in \mathcal{B}_{Q}$ :

- $\phi_{g}^{1}\left(<\Delta_{G}, p>\right)=\phi_{g}^{1}(G \cap p)=g^{-1}(G) \cap g^{-1}(p)=<\Delta_{g^{-1}(G)}, g^{-1}(p)>=$

- $\phi_{g}^{1}(<G \times\{y\}, p>)=\left\{\begin{array}{cl}g^{-1}(G) & \text { if } y \in p \\ \emptyset & \text { otherwise }\end{array}\right.$ $=<\phi_{g}^{2}\left(\Delta_{G}\right), \phi_{g}^{1}(p)>$. $<\phi_{g}^{2}(G \times\{y\}), \phi_{g}^{1}(p)>=<g^{-1}(G) \times\{y\}, g^{-1}(p)>=\left\{\begin{array}{cl}g^{-1}(G) & \text { if } y \in g^{-1}(p) \\ \emptyset & \text { otherwise }\end{array}\right.$

and then the conclusion follows from $(*)$.

PROPOSITION 5.7. $\phi$ is an embedding.

Proof. It is trivial to show that $\phi$ is a one-to-one functor on objects. In order to show that $\phi$ is also faithful consider

$$
g, g^{\prime}: Q^{\prime} \longrightarrow Q
$$

non-constant continuous mappings such that $g \neq g^{\prime}$. So, there exists $z \in Q^{\prime}$ such that $g(z) \neq g^{\prime}(z)$. Since $Q$ is Hausdorff there are opens $U, U^{\prime}$ in $\mathcal{T}$ verifying,

$$
g(z) \in U, g^{\prime}(z) \in U^{\prime} \text { and } U \cap U^{\prime}=\emptyset
$$

that is,

$$
z \in \phi_{g}^{1}(U), z \notin \phi_{g^{\prime}}^{1}(U)
$$

Consequently, $\phi(g) \neq \phi\left(g^{\prime}\right)$. 


\section{The embedding is full}

This section is devoted to the proof of the fullness of the functor $\phi$ constructed in the previous section. The proof is still rather involving. We maintain the notation of the previous section.

Let

$$
\left(h_{1}, h_{2}\right):\left(\mathcal{B}_{Q}, \mathcal{A}_{Q}\right) \longrightarrow\left(\mathcal{B}_{Q^{\prime}}, \mathcal{A}_{Q^{\prime}}\right)
$$

be a homomorphism of Kripke structures. We are going to construct a non-constant continuous mapping $g: Q^{\prime} \longrightarrow Q$ and show that g satisfies $\phi(g)=\left(h_{1}, h_{2}\right)$.

Let $x \in Q^{\prime}$. Consider

$$
\mathcal{F}_{x}=\left\{F \in \mathcal{B}_{Q}: F \text { is closed in } Q \text { and } x \in h_{1}(F)\right\} .
$$

We have

(i) $F \in \mathcal{F}_{x} \Longrightarrow F \neq \emptyset$;

(ii) $F_{1}, F_{2} \in \mathcal{F}_{x} \Longrightarrow F_{1} \cap F_{2} \in \mathcal{F}_{x}$;

(iii) $I_{x}=\bigcap\left\{F: F \in \mathcal{F}_{x}\right\} \neq \emptyset$ (it follows from the compactness of $Q$, (ii) and (i));

(iv) $I_{x}$ contains one element only.

We prove (iv). If there are $z_{1}, z_{2} \in I_{x}$ with $z_{1} \neq z_{2}$, then there are $U_{1}, U_{2} \in \mathcal{T}$ such that

$$
z_{1} \in U_{1}, z_{2} \in U_{2} \text { and } U_{1} \cap U_{2}=\emptyset .
$$

Thus, $h_{1}\left(U_{1}\right) \cap h_{1}\left(U_{2}\right)=\emptyset$, since $h_{1}$ is a Boolean homomorphism.

Therefore, if $x \in h_{1}\left(U_{1}\right)$ we would have $x \notin h_{1}\left(U_{2}\right)$, i.e., $x \in h_{1}\left(U_{2}^{c}\right)$. It would then follow $U_{2}^{c} \in \mathcal{F}_{x}$ and $z_{2} \in U_{2}^{c}$, which is a contradiction.

If $x \notin h_{1}\left(U_{1}\right)$ we would reach a contradiction in the same way.

Thus, we define $g(x)$ to be the unique element of the set $I_{x}$. Let us write

$$
g(x)=\bigcap_{F \in \mathcal{F}_{x}} F .
$$

LEMMA 6.1. $g$ is a non-constant mapping.

Proof. We have,

$$
<Q \times\left\{y_{1}\right\},\left\{y_{1}\right\}>=Q \text { and }<Q \times\left\{y_{2}\right\},\left\{y_{2}\right\}>=Q .
$$

Applying the homomorphism $h$ to these equalities, necessarily we conclude

$$
h_{1}\left(\left\{y_{1}\right\}\right) \neq \emptyset \text { and } h_{1}\left(\left\{y_{2}\right\}\right) \neq \emptyset \text {. }
$$

Taking one element $x_{1}$ in the first set and one element $x_{2}$ in the second set, we get $g\left(x_{1}\right)=y_{1} \neq y_{2}=g\left(x_{2}\right)$.

LEMMA 6.2. The mapping $g$ is continuous.

Proof. The conclusion comes after the following sub-lemmas 6.3-6.7. 
LEMMA 6.3. If $U \in \mathcal{T}$ then $h_{1}(U)$ is open in $Q^{\prime}$.

Proof. If $U \in \mathcal{T}$ we have $\Delta_{U} \in \mathcal{A}_{Q}$. Thus,

$$
<\Delta_{U}, U>=U \text { and }<\Delta_{U}, U^{c}>=\emptyset .
$$

Applying the homomorphism $h$ we conclude

$$
(*)<h_{2}\left(\Delta_{U}\right), h_{1}(U)>=h_{1}(U) \text { and }(* *)<h_{2}\left(\Delta_{U}\right),\left(h_{1}(U)\right)^{c}>=\emptyset \text {. }
$$

Besides,

$$
h_{2}\left(\Delta_{U}\right)=\Delta_{G} \cup G_{1} \times\left\{y_{1}\right\} \cup G_{2} \times\left\{y_{2}\right\}
$$

for some open sets $G, G_{1}, G_{2} \in \mathcal{B}_{\mathcal{T}^{\prime}}$.

Then, $\left({ }^{* *}\right)$ yields

$$
<\Delta_{G} \cup G_{1} \times\left\{y_{1}\right\} \cup G_{2} \times\left\{y_{2}\right\},\left(h_{1}(U)\right)^{c}>=\emptyset .
$$

Thus,

$$
\begin{gathered}
G \cap\left(h_{1}(U)\right)^{c}=\emptyset, \\
G \subseteq h_{1}(U) .
\end{gathered}
$$

On the other hand, $(*)$ gives, successively,

$$
\begin{gathered}
<\Delta_{G} \cup G_{1} \times\left\{y_{1}\right\} \cup G_{2} \times\left\{y_{2}\right\}, h_{1}(U)>=h_{1}(U), \\
\underbrace{\left(G \cap h_{1}(U)\right)}_{G} \cup\left(G_{1} \cap \delta_{y_{1}}^{h_{1}(U)}\right) \cup\left(G_{2} \cap \delta_{y_{2}}^{h_{1}(U)}\right)=h_{1}(U) .
\end{gathered}
$$

Consequently, $h_{1}(U)$ is open.

LEMMA 6.4. For $U \in \mathcal{T}$ it holds:

(i) $\operatorname{ext}_{Q}(U)$ is a finite union of elements of $\mathcal{T}$;

(ii) $\bar{U} \in \mathcal{B}_{Q}$;

(iii) $h_{1}(\bar{U})$ is closed in $Q^{\prime}$.

Proof. (ii) Follows immediately from (i).

(iii) By (i) there are $U_{1}, \ldots, U_{n} \in \mathcal{T}$ such that

$$
\operatorname{ext}(U)=\bigcup_{i=1}^{n} U_{i}
$$

Therefore

$$
h_{1}(\bar{U})=h_{1}\left(\operatorname{ext}(U)^{c}\right)=\left(\bigcup_{i=1}^{n} h_{1}\left(U_{i}\right)\right)^{c} .
$$

The conclusion follows from Lemma 6.3.

(i) Firstly, we decompose each space $A, B, C$ and $H^{X}$ in some finite union of regularly open sets in the respective space. Then, $Q$ will be a finite union of elements of the basis by means of those decompositions.

Since $A$ is Hausdorff and regular there are regularly open sets $U_{A}^{0}$ and $U_{A}^{1}$ in $A$ such that

$$
a_{0} \in U_{A}^{0}, a_{1} \in U_{A}^{1} \text { and } U_{A}^{0} \cap U_{A}^{1}=\emptyset .
$$


Thus, $U_{A}^{0} \subseteq\left(U_{A}^{1}\right)^{c}$ and since $U_{A}^{1}$ is open, we get $c l_{A}\left(U_{A}^{0}\right) \subseteq\left(U_{A}^{1}\right)^{c}$. Therefore, $a_{1} / \in c l_{A}\left(U_{A}^{0}\right)$ and the same applying to $a_{0}$. Now, by regularity there are regularly open sets $G^{0}$ and $G^{1}$ in $A$ such that

$$
\begin{aligned}
& a_{0} \in G^{0} \subseteq c l_{A}\left(G^{0}\right) \subseteq U_{A}^{0}, \\
& a_{1} \in G^{1} \subseteq c l_{A}\left(G^{1}\right) \subseteq U_{A}^{1} .
\end{aligned}
$$

The exterior of regularly open sets is still regularly open. Denoting by $U_{A}^{2}$ the set $\operatorname{ext}_{A}\left(G^{0}\right) \bigcap \operatorname{ext}_{A}\left(G^{1}\right)$, which is still regularly open, we have

$$
A=U_{A}^{0} \cup U_{A}^{1} \cup U_{A}^{2} .
$$

The same applying to $B$ and $C$.

Since $\{0,1\}^{X}$ is a closed set, the same reasoning allows us to choose in $\mathcal{T}_{0}$ regularly open neighborhoods $U_{H^{X}}^{a}$ and $U_{H^{X}}^{b}$ of $c_{a}$ and $c_{b}$, respectively, in such a way that they have their closure disjoint of $\{0,1\}^{X}$ and moreover, $c_{a} \notin c l_{H^{X}}\left(U_{H^{X}}^{b}\right), c_{b} \notin c l_{H^{X}}\left(U_{H^{X}}^{a}\right)$. Then, it is possible to choose $U_{H^{X}}^{\chi}=\prod_{x \in X} U_{x} \in \mathcal{T}_{0}$ such that $\chi_{R} \subseteq U_{H^{X}}^{\chi}$, (thus $\left.c l_{H^{X}}\left(U_{H^{X}}^{\chi}\right) \cap \chi_{R}=U_{H^{X}}^{\chi} \cap \chi_{R}\right), c_{a}, c_{b} \notin c l_{H^{X}}\left(U_{H^{X}}^{\chi}\right)$ and

$$
H^{X}=U_{H^{X}}^{a} \cup U_{H^{X}}^{b} \cup U_{H^{X}}^{\chi} .
$$

Actually, as $U_{H^{X}}^{a} \cup U_{H^{X}}^{b}$ is strictly contained in $H^{X}$, there exists $x_{0} \in X$ such that $U_{x_{0}}^{a} \cup U_{x_{0}}^{b}$ is strictly contained in $H$ as well, where $U_{x_{0}}^{a}$ and $U_{x_{0}}^{b}$ are the $x_{0}$-factors of $U_{H^{X}}^{a}$ and $U_{H^{X}}^{b}$, respectively. Then, it is enough to consider in $\prod_{x \in X} U_{x}$ all the $U_{x}$ 's equal to $H$, but $U_{x_{0}}$. As it was made for the space $A, U_{x_{0}}$ can be chosen such that

$$
H=U_{x_{0}}^{a} \cup U_{x_{0}}^{b} \cup U_{x_{0}}
$$

and $a, b \notin c l_{H}\left(U_{x_{0}}\right)$.

We decompose $\chi_{R} \times C$ by means of the corresponding decomposition of $C=U_{C}^{0} \cup U_{C}^{1} \cup U_{C}^{2}$ :

$$
\chi_{R} \times C=\left(\chi_{R} \times U_{C}^{0}\right) \cup\left(\chi_{R} \times U_{C}^{1}\right) \cup\left(\chi_{R} \times U_{C}^{2}\right) .
$$

Given $U \in \mathcal{T}$, we find a finite decomposition of $\operatorname{ext}_{Q}(U)$ in terms of the elements of $\mathcal{T}$, after replacing $A, B, H^{X}$ and $\chi_{R} \times C$ in the following expression by their decompositions:

$$
\operatorname{ext}(U)=(\operatorname{ext}(U) \cap A) \cup(\operatorname{ext}(U) \cap B) \cup\left(\operatorname{ext}(U) \cap H^{X}\right) \cup\left(\operatorname{ext}(U) \cap\left(\chi_{R} \times C\right)\right)
$$

Denote by $\mathcal{R}_{\mathcal{T}}$ the family of open sets of $Q$ which are finite unions of open sets of the basis $\mathcal{T}$.

LEMMA 6.5. For $G_{1} \in \mathcal{R}_{\mathcal{T}}$ and $G_{2} \in \mathcal{B}_{Q}$, if $\overline{G_{1}} \subseteq G_{2}$ then $\overline{h_{1}\left(G_{1}\right)} \subseteq h_{1}\left(G_{2}\right)$.

Proof. Let $G_{1} \in \mathcal{T}$, since the conclusion about the more general case follows immediately from this one.

Thus, the monotony of $h_{1}$ yields

$$
h_{1}\left(G_{1}\right) \subseteq h_{1}\left(\overline{G_{1}}\right) \text { and } h_{1}\left(\overline{G_{1}}\right) \subseteq h_{1}\left(G_{2}\right) .
$$

The required inclusion holds since $h_{1}\left(\overline{G_{1}}\right)$ is closed.

The following Lemma is valid in every regular compact space. 
LEMMA 6.6. For every closed $F$ in $Q$ we have

$$
F=\bigcap_{\left\{G \in \mathcal{R}_{\mathcal{T}}: G \supseteq F\right\}} \bar{G} .
$$

Proof. The inclusion $(\subseteq)$ is obvious. Suppose that it is a strict one. Let $x$ be one element of the intersection which doesn't belong to $F$. By regularity, there are open sets $V_{1}, V_{2}$ such that

$$
x \in V_{1}, F \subseteq V_{2} \text { and } V_{1} \cap V_{2}=\emptyset .
$$

For each $y \in F$ there exists $U_{y} \in \mathcal{T}$ such that $y \in U_{y} \subseteq V_{2}$. F is compact, so there exists a finite union of those $U_{y}$, let's say $G_{2}$, satisfying $F \subseteq G_{2} \subseteq V_{2}$. The hypothesis implies $x \in \overline{G_{2}}$. Now, we have successively,

$V_{1} \cap V_{2}=\emptyset \Longrightarrow V_{2} \subseteq V_{1}^{c} \Longrightarrow \overline{G_{2}} \subseteq V_{1}^{c} \Longrightarrow x \in V_{1}^{c}$, which is a contradiction.

LEMMA 6.7. For every closed $F$ in $Q$ the following equalities hold

$$
g^{-1}(F)=\bigcap_{F \subseteq G \in \mathcal{R}_{\mathcal{T}}} h_{1}(G)=\bigcap_{F \subseteq G \in \mathcal{R}_{\mathcal{T}}} \overline{h_{1}(G)} .
$$

Proof. We begin with the proof of the second equality. The inclusion $(\subseteq)$ is obvious. For the proof of the other inclusion we consider $G \in \mathcal{R}_{\mathcal{T}}$ such that $F \subseteq G$. $Q$ is a normal space, so there exists an open $U^{\prime}$ such that

$$
F \subseteq U^{\prime} \subseteq \overline{U^{\prime}} \subseteq G
$$

By an argument similar to the one used to prove the last Lemma, there exists $U \in \mathcal{R}_{\mathcal{T}}$ such that

$$
F \subseteq U \subseteq U^{\prime}
$$

Consequently,

$$
F \subseteq U \subseteq \bar{U} \subseteq G
$$

By Lemma 6.5 we have $\overline{h_{1}(U)} \subseteq h_{1}(G)$.

Next, we prove the first equality.

$(\subseteq)$ Let $G \in \mathcal{R}_{\mathcal{T}}$ such that $F \subseteq G$. Then, $G^{c}$ is closed and still belongs to $\mathcal{B}_{Q}$. By definition of $g$ we write $x \in h_{1}\left(G^{c}\right) \Longrightarrow g(x) \in G^{c}$. That is, $h_{1}\left(G^{c}\right) \subseteq g^{-1}\left(G^{c}\right)$, which is the same as $g^{-1}(G) \subseteq h_{1}(G)$, since $h_{1}$ is a Boolean homomorphism. From this together with $F \subseteq G$, we conclude

$$
g^{-1}(F) \subseteq h_{1}(G)
$$

as it was required.

() Let $x \in \bigcap_{F \subseteq G \in \mathcal{R}_{\mathcal{T}}} h_{1}(G)$. Thus, for every $G \in \mathcal{R}_{\mathcal{T}}$, we have

$$
F \subseteq G \Longrightarrow x \in h_{1}(G) \subseteq h_{1}(\bar{G})
$$

It follows $g(x) \in \bar{G}$, then by Lemma $6.6, g(x) \in F$, that is, $x \in g^{-1}(F)$.

REMARK 6.8. If $g: Q^{\prime} \longrightarrow Q$ is a non-constant continuous mapping, we can guarantee the existence of a homomorphism of graphs

$$
f:(X, R) \longrightarrow\left(X^{\prime}, R^{\prime}\right)
$$

such that $\psi(f)=g$. Therefore, we know the form of $g$. 
The following proposition will complete the establishment of the fullness.

PROPOSITION 6.9. $\phi(g)=\left(h_{1}, h_{2}\right)$.

Proof. To conclude that $\phi_{g}^{1}=h_{1}$ is enough to prove that $\phi_{g}^{1}(U)=h_{1}(U)$, for every $U \in \mathcal{T} \cup\left\{\left\{y_{1}\right\},\left\{y_{2}\right\}\right\}$, since this set generates $\mathcal{B}_{Q}$. The proof of this fact is finished after the Lemmas 6.10-6.14.

LEMMA 6.10. It holds $h_{1}\left(\left\{y_{1}\right\}\right)=g^{-1}\left(\left\{y_{1}\right\}\right)$ and $h_{1}\left(\left\{y_{2}\right\}\right)=g^{-1}\left(\left\{y_{2}\right\}\right)$.

Proof. We have $<Q \times\left\{y_{1}\right\},\left\{y_{1}\right\}>=Q$. Applying $h_{1}$, it follows that

$$
<h_{2}\left(Q \times\left\{y_{1}\right\}\right), h_{1}\left(\left\{y_{1}\right\}\right)>=Q^{\prime},
$$

and so $h_{1}\left(\left\{y_{1}\right\}\right) \neq \emptyset$.

On the other hand, by definition of $g, y \in h_{1}\left(\left\{y_{1}\right\}\right) \Longrightarrow g(y)=y_{1}$. Remark 6.8 implies $y=y_{1}$. Therefore,

$$
h_{1}\left(\left\{y_{1}\right\}\right)=g^{-1}\left(\left\{y_{1}\right\}\right)
$$

Similarly for $y_{2}$.

Now, we have to prove that $h_{1}(U)=g^{-1}(U)$ for $U \in \mathcal{T}$. The definition of $g$ yields $h_{1}(F) \subseteq g^{-1}(F)$ for every closed $F \in \mathcal{B}_{Q}$.

So, we can write immediately

$$
g^{-1}(U) \subseteq h_{1}(U) \subseteq h_{1}(\bar{U}) \subseteq g^{-1}(\bar{U}) .
$$

If we show that $\operatorname{int}\left(g^{-1}(\bar{U})\right) \subseteq g^{-1}(U)$, then we will be able to conclude the required equality, since $h_{1}(U)$ is open (Lemma 6.3 ).

LEMMA 6.11. For $U \in \mathcal{T}$, we have

$$
\operatorname{int}\left(g^{-1}(\bar{U})\right) \subseteq g^{-1}(U) .
$$

Proof. We consider the proof for $U \in \mathcal{T}_{7}$, the other cases being similar or straightforward. Note that $g=\psi(f)$ for some graph homomorphism $f$.

We begin with the calculation of $g^{-1}\left(\overline{\prod_{x \in X} U_{x}}\right)$ for $\prod_{x \in X} U_{x} \in \mathcal{T}_{0}$. As in the proof of Lemma $5.5($ ii), we can write

$$
g^{-1}\left(\overline{\prod_{x \in X} U_{x}}\right)=g^{-1}\left(\prod_{x \in X} c l_{H}\left(U_{x}\right)\right)=\prod_{x^{\prime} \in X^{\prime}} V_{x^{\prime}}, \text { where } V_{x^{\prime}}=\bigcap_{x \in f^{-1}\left(\left\{x^{\prime}\right\}\right)} c l_{H}\left(U_{x}\right) .
$$

Now, if $U=\left(\prod_{x \in X} U_{x}\right) \cup\left(\left(\prod_{x \in X} U_{x}\right) \cap \chi_{R}\right) \times U_{C} \in \mathcal{T}_{7}$, we have

$$
\bar{U}=\left(\prod_{x \in X} c l_{H}\left(U_{x}\right)\right) \cup c l \chi_{R}\left(\left(\prod_{x \in X} U_{x}\right) \cap \chi_{R}\right) \times c l_{C}\left(U_{C}\right) .
$$

Since $U \in \mathcal{T}_{7}$, the set $\left(\prod_{x \in X} U_{x}\right) \cap \chi_{R}$ is closed in $\chi_{R}$. 
Therefore,

$$
g^{-1}(\bar{U})=\left(\prod_{x^{\prime} \in X^{\prime}} V_{x^{\prime}}\right) \cup\left(\left(\prod_{x^{\prime} \in X^{\prime}} U_{x^{\prime}}^{\prime}\right) \cap \chi_{R^{\prime}}\right) \times c l_{C}\left(U_{C}\right)
$$

where

$$
U_{x^{\prime}}^{\prime}=\bigcap_{x \in f^{-1}\left(\left\{x^{\prime}\right\}\right)} U_{x}
$$

Since

$$
c l_{H^{X}}\left(\left(\prod_{x^{\prime} \in X^{\prime}} U_{x^{\prime}}^{\prime}\right) \cap \chi_{R}\right)=\left(\prod_{x^{\prime} \in X^{\prime}} V_{x^{\prime}}\right) \cap \chi_{R^{\prime}}=\left(\prod_{x^{\prime} \in X^{\prime}} U_{x^{\prime}}^{\prime}\right) \cap \chi_{R^{\prime}},
$$

and each $V_{x^{\prime}}$ is, effectively, a finite intersection, we have, successively,

$$
\begin{aligned}
& \operatorname{int}\left(g^{-1}(\bar{U})\right)= \\
= & \left(\prod_{x^{\prime} \in X^{\prime}} i n t_{H}\left(V_{x^{\prime}}\right)\right) \cup i n \chi_{R^{\prime}}\left(\left(\prod_{x^{\prime} \in X^{\prime}} U_{x^{\prime}}^{\prime}\right) \cap \chi_{R^{\prime}}\right) \times i n t_{n}\left(c l_{C}\left(U_{C}\right)\right)= \\
= & \left(\prod_{x^{\prime} \in X^{\prime}} U_{x^{\prime}}^{\prime}\right) \cup\left(\left(\prod_{x^{\prime} \in X^{\prime}} U_{x^{\prime}}^{\prime}\right) \cap \chi_{R^{\prime}}\right) \times U_{C}=g^{-1}(U)
\end{aligned}
$$

To finalize the proof of the fullness it remains to prove

$$
\phi_{g}^{2}=h_{2}
$$

It is sufficient to show that

$$
h_{2}\left(\Delta_{U}\right)=\Delta_{g^{-1}(U)}, \quad h_{2}\left(Q \times\left\{y_{1}\right\}\right)=Q^{\prime} \times\left\{y_{1}\right\} \quad \text { and } \quad h_{2}\left(Q \times\left\{y_{2}\right\}\right)=Q^{\prime} \times\left\{y_{2}\right\}
$$

since those binary relations generate $\mathcal{A}_{Q}$.

We begin with the proof of a special case, which will be useful to solve the other cases.

LEMMA 6.12. $h_{2}\left(\Delta_{Q}\right)=\Delta_{Q^{\prime}}$.

Proof. Let's suppose that $h_{2}\left(\Delta_{Q}\right) \nsubseteq \Delta_{Q^{\prime}}$. Then, there exists $x, y \in Q^{\prime}$, with $x \neq y$, such that $(x, y) \in h_{2}\left(\Delta_{Q}\right)$. Necessarily, $y \in\left\{y_{1}, y_{2}\right\}$.

Applying the homomorphism $h$ to $<\Delta_{Q},\{y\}>=\{y\}$, we have

$$
<h_{2}\left(\Delta_{Q}\right), h_{1}(\{y\})>=h_{1}(\{y\}) .
$$

Since $h_{1}(\{y\})=\{y\}$, it follows that

$$
x \in<h_{2}\left(\Delta_{Q}\right),\{y\}>=\{y\}
$$

which is a contradiction. Consequently, $h_{2}\left(\Delta_{Q}\right) \subseteq \Delta_{Q^{\prime}}$, i.e.,

$$
h_{2}\left(\Delta_{Q}\right)=\Delta_{G} \text { for some subset } G \subseteq Q^{\prime} .
$$

But, from $<\Delta_{Q}, Q>=Q$, we have, successively,

$$
<\underbrace{h_{2}\left(\Delta_{Q}\right)}_{\Delta_{G}}, \underbrace{h_{1}(Q)}_{Q^{\prime}}>=\underbrace{h_{1}(Q)}_{Q^{\prime}} \Longrightarrow G \cap Q^{\prime}=Q^{\prime} \Longrightarrow G=Q^{\prime} .
$$

LEMMA 6.13. Let $\Delta_{G} \in \mathcal{A}_{Q}$. Then $h_{2}\left(\Delta_{G}\right)=\Delta_{g^{-1}(G)}$. 
Proof. We have $\Delta_{Q}=\left(\Delta_{G}\right)^{*}$. Therefore $h_{2}\left(\Delta_{Q}\right)=\left(h_{2}\left(\Delta_{G}\right)\right)^{*}$, that is,

$$
h_{2}\left(\Delta_{Q}\right)=\Delta_{Q^{\prime}} \cup h_{2}\left(\Delta_{G}\right) \cup\left(h_{2}\left(\Delta_{G}\right) ; h_{2}\left(\Delta_{G}\right)\right) \cup \ldots
$$

Then $h_{2}\left(\Delta_{G}\right) \subseteq h_{2}\left(\Delta_{Q}\right)=\Delta_{Q^{\prime}}$. Thus, $h_{2}\left(\Delta_{G}\right)=\Delta_{G^{\prime}}$, for some $G^{\prime} \subseteq Q^{\prime}$. Applying $h$ to $<\Delta_{G}, Q>=G$ we get

$$
<\Delta_{G^{\prime}}, Q^{\prime}>=h_{1}(G) .
$$

Consequently, $G^{\prime}=h_{1}(G)=g^{-1}(G)$.

LEMMA 6.14. It holds $h_{2}\left(Q \times\left\{y_{1}\right\}\right)=Q^{\prime} \times\left\{y_{1}\right\}$ and $h_{2}\left(Q \times\left\{y_{2}\right\}\right)=Q^{\prime} \times\left\{y_{2}\right\}$.

Proof. Applying $h$ to $<Q \times\left\{y_{1}\right\},\left\{y_{1}\right\}>=Q$ we can write

$$
<h_{2}\left(Q \times\left\{y_{1}\right\}\right),\left\{y_{1}\right\}>=Q^{\prime}
$$

due to Lemma 6.10. Then $Q^{\prime} \times\left\{y_{1}\right\} \subseteq h_{2}\left(Q \times\left\{y_{1}\right\}\right)$.

Similarly,

$$
<Q \times\left\{y_{1}\right\},\left\{y_{1}\right\}^{c}>=\emptyset \Longrightarrow<h_{2}\left(Q \times\left\{y_{1}\right\}\right),\left(\left\{y_{1}\right\}\right)^{c}>=\emptyset
$$

Consequently,

$$
\left(Q^{\prime} \times\left(\left\{y_{1}\right\}^{c}\right)\right) \cap h_{2}\left(Q \times\left\{y_{1}\right\}\right)=\emptyset .
$$

Thus, we can finally conclude that

$$
h_{2}\left(Q \times\left\{y_{1}\right\}\right)=Q^{\prime} \times\left\{y_{1}\right\} .
$$

Mutatis mutandis for $y_{2}$.

\section{Applications}

Now, we list some of the consequences of the alg-universality of SKri .

COROLLARY 7.1. The category SDA is alg-universal.

COROLLARY 7.2. Any monoid is isomorphic to the endomorphism monoid of some separable Kripke structure.

Proof. This result is due to the fact that every small category is algebraic. Thus, it is enough to fully embed in SKri the small category with one object and having the given monoid as its endomorphism monoid.

COROLLARY 7.3. For every group $G$ there exists a separable Kripke structure $K$ such that $G \cong \operatorname{Aut}(K)=\operatorname{End}(K)$.

Proof. Consider the small category with one object and having $G$ as its set of endomorphisms. Notice that every endomorphism in this category is an isomorphism.

The following property is established in every algebraic and algebraically universal category as it was proved by Hedrlín and Sichler [12].

COROLLARY 7.4. SKri contains a proper class of mutually disjoint copies of itself. 
It was independently proved by Magill [24], Maxson [25] and Schein [36] that a Boolean algebra is uniquely determined by its endomorphism monoid (that is, for Boolean algebras $B, B^{\prime}$, if $\operatorname{End}(B) \cong \operatorname{End}\left(B^{\prime}\right)$ then $\left.B \cong B^{\prime}\right)$. This property is not shared by the objects of alg-universal categories as we show bellow.

COROLLARY 7.5. For any monoid $M$ and for every cardinal $n$, there exists a family of separable Kripke structures $\left(K_{i}\right)_{i<n}$ with $\operatorname{End}\left(K_{i}\right) \cong M$ and $\operatorname{Hom}\left(K_{i}, K_{j}\right)=\emptyset$ for distinct $i, j<n$.

Proof. It is enough to fully embed in $S K r i$ a small category $\mathcal{S}$ with $n$ objects and with morphisms

$$
\mathcal{S}(i, j)= \begin{cases}M & \text { if } i=j \\ \emptyset & \text { otherwise }\end{cases}
$$

for objects $i$ and $j$ of $\mathcal{S}$ (composition in $\mathcal{S}$ is defined according to the product in the monoid $M)$.

COROLLARY 7.6. Let $(I, \leq)$ be a poset and let $\left(G_{i}\right)_{i \in I}$ be a family of groups. Then, there exists a set $S$ and a family $\left(K_{i}\right)_{i \in I}$ of separable Kripke structures on $S$, such that $G_{i} \cong A u t\left(K_{i}\right)$, for $i \in I$. Moreover, a Kripke structure $K_{i}$ is a subalgebra of $K_{j}$ iff $i \leq j$.

Proof. The statement above is, once more, due to the possibility of fully embedding a convenient small category $\mathcal{S}$ in $S K r i$.

The construction of $\mathcal{S}$ is as follows. We suppose that there exists the meet $i \wedge i^{\prime}$ of each pair $i, i^{\prime} \in(I, \leq)$ and that there exists the maximum 1 of $(I, \leq)$ as well. Otherwise, the set $I$ is enlarged, and the corresponding new groups $G_{i}$ 's are taken arbitrarily. Thus, we define

$$
\operatorname{Obj} \mathcal{S}=I
$$

and for every $i, i^{\prime} \in I$ the set $\mathcal{S}\left(i, i^{\prime}\right)$ of all morphisms of $\mathcal{S}$ from $i$ to $i^{\prime}$ is

$$
\mathcal{S}\left(i, i^{\prime}\right)=\left\{\left(\rho_{i j}, g, \gamma_{j i^{\prime}}\right): j \in I, j \leq i \wedge i^{\prime}, g \in G_{j}\right\}
$$

where $\rho_{i j}$ and $\gamma_{j i^{\prime}}$ are formal symbols making the set of morphisms disjoint for different pairs of objects in order to have the domain and the codomain of an object in $\mathcal{S}$ correctly defined. The composition of morphisms in $\mathcal{S}$ (which is written, for convenience, from the left to the right) is defined by

$$
\left(\rho_{i j}, g, \gamma_{j i^{\prime}}\right) \circ\left(\rho_{i^{\prime} j^{\prime}}, g^{\prime}, \gamma_{j^{\prime} i^{\prime \prime}}\right)=\left\{\begin{array}{lll}
\left(\rho_{i j}, g \cdot g^{\prime}, \gamma_{j i^{\prime \prime}}\right) & \text { if } & j=\left(j \wedge j^{\prime}\right)=j^{\prime} \\
\left(\rho_{i j}, g, \gamma_{j i^{\prime \prime}}\right) & \text { if } & j=\left(j \wedge j^{\prime}\right) \neq j^{\prime} \\
\left(\rho_{i j^{\prime}}, g^{\prime}, \gamma_{j^{\prime} i^{\prime \prime}}\right) & \text { if } & j \neq\left(j \wedge j^{\prime}\right)=j^{\prime} \\
\left(\rho_{i j_{0}}, 1, \gamma_{j 0} i^{\prime}\right) & \text { if } & j_{0}=\left(j \wedge j^{\prime}\right), j_{0} \neq j, j_{0} \neq j^{\prime}
\end{array}\right.
$$

where $g \in G_{j}, g^{\prime} \in G_{j^{\prime}}$ and 1 is the unit of $G_{j_{0}}$. It is routine to check the associativity of the composition, hence $\mathcal{S}$ is really a category. This category was defined in [16].

For every pair $i, j \in I$, with $i \leq j$, let us denote $c_{i j}=\left(\rho_{i i}, 1, \gamma_{i j}\right)$, (where 1 is the unit of the group $\left.G_{i}\right)$ and $r_{j i}=\left(\rho_{j i}, 1, \gamma_{i i}\right)$. Then, for every $i, r_{i i}=c_{i i}$ is the identity $1_{i}$. For the sake of simplicity, we denote by $g\left(\in G_{i}\right)$ the morphism $\left(\rho_{i i}, g, \gamma_{i i}\right)$. Thus, each morphism $\left(\rho_{i j}, g, \gamma_{j i^{\prime}}\right)$ is the result of the composition $r_{i j} \circ g_{\circ} c_{j i^{\prime}}$.

$\left(^{*}\right)$ For each $i \leq j, c_{i j}$ is a monomorphism (moreover, since $c_{i j} \circ r_{j i}=1_{i}$, each $c_{i j}$ is a coretraction and $r_{j i}$ is the correspondent retraction). Besides, whenever $i \not \leq j$, there are no monomorphisms in $\mathcal{S}(i, j)$ since every morphism in $\mathcal{S}(i, j)$ is the composition of $r_{i, i \wedge j} \circ c_{i \wedge j, i}$ with itself. 
$\left({ }^{* *}\right)$ Every $g \in G_{i}$, seen as a morphism in $\mathcal{S}(i, i)$ is an isomorphism, and there is no other isomorphism in $\mathcal{S}(i, i)$.

Let $F: \mathcal{S} \longrightarrow S K r i$ be a full embedding. Then, $K_{1}=F(1)$ is a separable Kripke structure on a set, let's say, $S$. Consider, for each $i \in I$, the separable Kripke structure $K_{i}=\left(F\left(c_{i 1}\right)\right)(F(i))$ on $S$, which is the image of $F(i)$ under the monomorphism $F\left(c_{i 1}\right)$.

The conclusion follows from remarks $(*)$ and $(* *)$.

Recalling the condition (M) referred in the section 3, since SKri is algebraic we can establish,

COROLLARY 7.7. SKri it is universal iff (M) happens.

Every class of finitary algebras closed under the formation of homomorphic images, subalgebras and products is a variety, that is, can be presented by a set of equations. This is the well known Birkhoff theorem. Therefore, a countable set of variables is enough to describe the equations satisfied by all the algebras of such classes of algebras.

Now, the question to be addressed is about the number of variables necessary to describe classes of finitary algebras presented by implications. Regarding the existing flexibility in the definition of the concepts used bellow, we follow [2].

To define implication we fix a type $\Omega$ of finitary algebras and a set $V$ of variables. So, a $V$-implication for $\Omega$-algebras is a formal expression

$$
\left\{t_{i}=t^{\prime}{ }_{i}\right\}_{i \in I} \Longrightarrow s=s^{\prime}
$$

where $I$ is an index set and all $t_{i}, t^{\prime}{ }_{i}, s$ and $s^{\prime}$ are terms of the absolutely free $\Omega$-algebra $\mathrm{V}^{\#}$ generated by $V$.

We say that an $\Omega$-algebra $A$ satisfies that implication if for each substitution of variables

$$
f: V \longrightarrow A
$$

we have $f^{\#}(s)=f^{\#}\left(s^{\prime}\right)$, whenever

$$
f^{\#}\left(t_{i}\right)=f^{\#}\left(t^{\prime}{ }_{i}\right) \text { for all } i \in I,
$$

where $f^{\#}: V^{\#} \longrightarrow A$ is the homomorphic extension of $f$.

Classes of algebras closed under subalgebras and products, which we will designate by SP-classes, are presented by implications. This is a Theorem of Mal'cev (cf. [2]). The set of premises of an implication can be infinite, which gives rise to the question whether the number of variables to describe the class of implications satisfied by a given SP-class of algebras is always bounded or not. If we define a quasivariety to be a class of similar algebras presented by a set of implications then the question is whether SP-classes are always quasivarieties. (Although, in [1] a quasivariety is defined to be an SP-class of similar algebras.). The answer turns out to be dependent of the set theory we live in.

The problem was solved by Adámek in [1] where he proved that each SP-class of algebras is a quasivariety iff the following condition is stated:

Vopěnka's Principle: every class of models of a first order theory in which no model has an embedding to another one is a set.

Vopěnka's Principle can be reformulated in terms of the category of graphs yielding:

The category of graphs does not have a discrete full subcategory whose objects form a proper class. 
Notice that, by Theorem 7.5, in every algebraically universal category there exists a discrete full subcategory whose objects form a set of prescribed cardinality.

In Bernays-Gödel set theory with the axiom of choice for classes (BGC) measurable cardinals do exist if Vopěnka's Principle holds. Therefore, the negation of Vopěnka's Principle is consistent with BGC set theory.

Simultaneously, Vopěnka's Principle is also consistent with BGC set theory if huge cardinals exist. For details see [2] and [15]. From the alg-universality of SKri another formulation of Vopěnka's Principle is possible:

COROLLARY 7.8. Vopěnka's Principle holds iff there is no discrete full subcategory of $S$ Kri whose objects form a proper class.

Moreover, as in [1] one can deduce,

COROLLARY 7.9. Under the negation of Vopěnka's Principle there exists an SP-class of Kripke structures which is not a quasivariety.

\section{Open problem on test algebras}

A test algebra is a dynamic algebra endowed with one more operation ([29]), the test:

$$
?: \mathcal{B} \longrightarrow \mathcal{A}
$$

and one more equation, namely

$$
<p ?, q>=p \wedge q .
$$

A Kripke test structure $(\mathcal{B}, \mathcal{A}, S)$ is a Kripke structure equipped with a binary relation $p ?=\{(s, s): s \in p\}$ for every $p \in \mathcal{B}$. In the presence of test, Kripke structures gain expressiveness, since the meaning of the programs if $p$ then a else $b$ and while $p$ do $a$ can be captured, respectively, by $(p ?) a \cup(\neg p ?) b$ and $((p ?) a)^{*}(\neg p$ ?). Some results on test algebras can be obtained with slight modifications on results on dynamic algebras. See, e.g. [32].

The open problem is "Is the category of test algebras and their homomorphisms alguniversal'?

\section{Acknowledgments}

I would like to thank Professor Verra Trnková for the suggestion of this problem as well as for invaluable advice and careful comments without which this result wouldn't be achieved. I also thank the Faculty of Mathematics and Physics of Charles University for its hospitality during part of the preparation of this work. Finally, I thank Professor Maria Teresa Martins for her kind remarks.

\section{References}

[1] J. Adámek, How many variables does a quasivariety need?, Algebra Universalis 27 (1990), 44-48.

[2] J. Adámek and J. Rosický, Locally presentable and accessible categories, London Math. Soc. Lect. Notes, Series 189, Cambridge University Press, Cambridge, 1994. 
[3] G. Birkoff, On groups of automorphisms (in Spanish), Revista Unión Math. Argentina $11,155-157$.

[4] G. Birkhoff and J. D. Lipson, Heterogeneous algebras, J. Combinatorial Theory 8 (1970), 115-133.

[5] S. Burris and H. P. Sankappanavar, A Course in Universal Algebra, SpringerVerlag, New York, 1981.

[6] H. Cook, Continua which admit only the identity mapping onto non-degenerate subcontinua, Fund. Math. 60 (1967), 241-249.

[7] R. Frucht, Herstellung von Graphen mit vorgegebener abstrakter Gruppe, Compos. Math. 6 (1938), 239-250.

[8] D. Gabbay, Axiomatizations of logics of programs, unpublished manuscript, BarIlan Univ. Ramat-Gan, Israel 1977.

[9] J. de Groot, Groups represented by homeomorphisms groups, Math. Annalen 138 (1959), 80-102.

[10] Z. Hedrlín, Extension of structures and full embeddings of categories, Actes du Congrès Internat. des Mathématiciens, Nice, 1970, tome 1, Gauthier-Villars, Paris, 1971, 319-322.

[11] Z. Hedrlín and A. Pultr, On full embeddings of categories of algebras, Illinois J. of Math. 10 (1966), 392-406.

[12] Z. Hedrlín and J. Sichler, Any boundable binding category contains a proper class of mutually disjoint copies of itself, Algebra Universalis 1 (1971), 97-103.

[13] L. Henkin, The logic of equality, Amer. Math. Monthly 84, 8 (1977), 597-612.

[14] J. R. Isbell, Adequate Categories, Illinois J. Math. 4 (1960), 541-552.

[15] T. Jech, Set Theory, Academic Press New York, 1978.

[16] M. Kallus and V. Trnková, Symmetries and Retracts of Quantum Logics , Int. Journal of Theoretical Physics, Vol. 26, No. 1 (1987), 1-9.

[17] D. Kozen, On the representation of dynamic algebras, Report RC7898, IBM Research Center, Yorktown Heights, New York, Oct. 1979.

[18] D. Kozen, On the representation of dynamic algebras II, Report RC8290, IBM Research Center, Yorktown Heights, New York, May 1980.

[19] D. Kozen, A representation Theorem for model of *-free PDL, Proceedings 7th Internat. Coll. on Automata, Languages and Programming (J. W. de Bakker and J. van Leeuwen, eds.), Lect. Notes in Comp. Sci. 85 (1980), 351-362.

[20] D. Kozen, On the duality of dynamic algebras and Kripke models, Proceedings Workshop on Logics of Programs (E. Engeler, ed.), Lect. Notes in Comp. Sci. 125 (1981), $1-11$.

[21] D. Kozen and J. Tiuryn, Logics of Programs, Handbook of Theoretical Computer Science (J. van Leeuwen, ed.), Elsevier Science Publishers B.V., 1990. 
[22] L. Kučera, Lectures from the theory of categories (Czech), Charles University 1970 - preprint.

[23] L. Kučera and A. Pultr, Non-algebraic concrete categories, J. Pure Appl. Alg. 3 (1973), 95-102.

[24] K. D. Magill, The semigroup of endomorphisms of a Boolean ring, Semigroup Forum 4 (1972), 411-416.

[25] C. J. Maxson, On semigroups of Boolean ring endomorphisms, Semigroup Forum 4 (1972), 78-82.

[26] R. McKenzie and J. D. Monk, On automorphism groups of Boolean Algebras, 10. Infinite and finite sets, Keszthély, Hungary, 1973.

[27] I. Németi, Dynamic algebras of programs, Proceedings Fundamentals of Computation Theory, Lect. Notes in Comp. Sci. 117 (1981), 281-290.

[28] R. Parikh, A completeness result for a propositional dynamic logic, Lect. Notes in Comp. Sci. 64 (1978), 403-415.

[29] V. R. Pratt, Dynamic Algebras: examples, constructions, applications, Technical Report MIT/Laboratory for Comp. Sci./TM-138, July 1979, also published in Studia Logica, Vol. 50 (3/4) (1992), 571-605.

[30] A. Pultr and V. Trnková, Combinatorial, algebraic and topological representations of groups, semigroups and categories, North Holland, Amsterdam, 1980.

[31] J. Reiterman and V. Trnková, Dynamic algebras which are not Kripke structures, Proceedings 9th Symp. on Math. Found. of Computer Science (P. Dembińsky, ed.), Lect. Notes in Comp. Sci. 88 (1980), 528-538.

[32] J. Reiterman and V. Trnková, Dynamic algebras with test, Journal of Comp. and System Sci. 35 (1987), 229-242.

[33] J. Reiterman and V. Trnková, Free Structures, Category Theory at Work (H. Herrlich and H.-E. Porst, eds.), Heldermann Verlag, Berlin, 1991.

[34] G. Sabidussi, Graphs with given infinite groups, Monatsh. Math. 64 (1960), 64-67.

[35] K. Segerberg, A completeness theorem in the Modal Logic of Programs, Notices of the AMS, Vol. 24 (6)(1977), A-552.

[36] B. M. Schein, Ordered sets, semilattices, distributive lattices and Boolean algebras with homomorphic endomorphism semigroups, Fund. Math. 68 (1970), 31-50.

[37] V. Trnková, Vse malyje kategorii predstavimy nepreryvnymi nepostojannymi otobraženijami bikompaktov, DAN SSSR 230 (1976), 789-791.

[38] W. Wechler, Universal Algebra for Computer Scientists, Springer Verlag, Berlin, 1992.

[39] S. Willard, General Topology, Addison-Wesley, London, 1970.

CMUC
Departamento de Matemática
Universidade de Coimbra
P-3000 COIMBRA
Portugal

